\title{
Chemoselective Cyclizations of Divinyl Ketones to Cyclohexenones Mediated by Lewis Acid and Base
}

\author{
Nabi A. Magomedov, Piero L. Ruggiero, and Yuchen Tang \\ Department of Chemistry, University of Rochester, Rochester, NY 14627-0216 \\ magomedov@chem.rochester.edu
}

\section{Supporting Information}

General Information. All reactions were carried out under an atmosphere of nitrogen in oven-dried glassware with magnetic stirring. Solvents and reagents were purified prior to use following the guidelines of Perrin and Armarego ${ }^{1}$, except for THF, toluene, acetonitrile, dichloromethane and diethylether, which were dispensed using the Glass Contour solvent purification system. Air- and moisture-sensitive liquids and solutions were transferred via syringe or stainless steel cannula. Organic solutions were concentrated in vacuo below $45^{\circ} \mathrm{C}$ at approximately $20 \mathrm{~mm} \mathrm{Hg}$. Purification of reaction products was carried out by flash chromatography using EM Reagent silica gel 60 (230-400 mesh). Analytical thin layer chromatography was performed on EM Reagent $0.25 \mathrm{~mm}$ silica gel $60-\mathrm{F}$ plates. Visualization was accomplished with UV light and potassium permanganate solution, ceric ammonium molybdate, or $p$-anisaldehyde solution followed by heating. Melting points were measured with a Bibby Sterilin Stuart SMP10 melting point apparatus and are uncorrected. Infrared spectra were recorded on a Perkin Elmer 1600 series FT-IR spectrometer. ${ }^{1} \mathrm{H}$ NMR spectra were recorded on a Bruker Avance-400 (400 MHz) or Bruker AMX-400 (400 MHz) spectrometer and are reported in ppm using the solvent as an internal standard $\left(\mathrm{CDCl}_{3}\right.$ at $\left.7.26 \mathrm{ppm}\right)$. Data are reported as $(\mathrm{br}=\mathrm{broad}, \mathrm{s}=$ singlet, $\mathrm{d}=$ doublet, $\mathrm{t}=$ triplet, $\mathrm{q}=$ quartet, $\mathrm{m}=$ multiplet; coupling constant(s) in Hz; integration). Proton-decoupled ${ }^{13} \mathrm{C}$ NMR spectra were recorded on a Bruker Avance-400 (100 MHz) or Bruker AMX-400 (100 MHz) spectrometer and are reported in ppm using the solvent as the internal standard $\left(\mathrm{CDCl}_{3}\right.$ at $\left.77.23 \mathrm{ppm}\right)$. High-resolution mass spectra were obtained on a Thermo Finnigan MAT 95 XL Magnetic Sector spectrometer with electrospray ionization at the State University of New York at Buffalo Mass Spectrometry Facility. trans-Styryl-p-tolyl sulfone, ${ }^{2}$ 1-benzenesulfonyl-4-methylpent-3en-2-one, ${ }^{3}$ phenyl-2,2-diphenylethenyl sulfone, ${ }^{4} \quad$ (1,7,7-trimethylbicycle[2.2.1]hept-2-ylidene)-

\footnotetext{
${ }^{1}$ Armarego, W. L. F.; Perrin, D. D. Purification of Laboratory Chemicals; Butterworth-Heinemann: Bath, 2002.

${ }^{2}$ Nair, V.; Augustine, A.; Suja, T. D. Synthesis 2002; 15, 2259-2265.

${ }^{3}$ Lansbury, P. T.; Spagnuolo, C. J.; Grimm, E. L. Tetrahedron Lett. 1986, 27, 2725-2726.
} 
acetaldehyde $^{5}$ cycloheptylidene acetaldehyde $^{6}$, and (3,3-dimethylbut-1-ene-1-sulfonyl)benzene ${ }^{7}$ were $^{-}$ prepared according to literature procedures. 3-Methylbut-2-enal, 3-phenylpropenal, trans-4methylpent-2-enal, benzaldehyde, methyl phenyl sulfone, and ethyl tiglate were purchased and used without further purification.

\section{Synthesis of Substrates for Chemoselective Cyclizations}

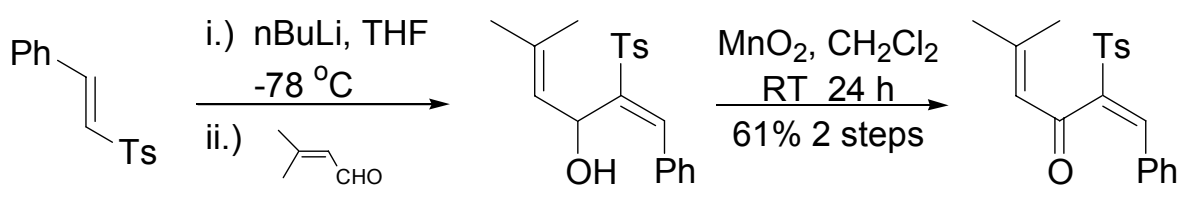

Procedure A. To a solution of $250 \mathrm{mg}(0.97 \mathrm{mmol})$ of trans-styryl-p-tolyl sulfone in $3.2 \mathrm{~mL}$ of dry THF, cooled to $-78^{\circ} \mathrm{C}$, was added dropwise $0.51 \mathrm{~mL}$ of a $2.0 \mathrm{M}$ solution of $n$-BuLi (1.0 mmol) in cyclohexane. After stirring for $15 \mathrm{~min}$ at the bath temperature, $85 \mathrm{mg}$ (98 $\mu \mathrm{L}, 1.0 \mathrm{mmol})$ of 3-methyl2-butenal was added quickly. The reaction mixture was stirred at $-78{ }^{\circ} \mathrm{C}$ for 15 minutes and then warmed to room temperature over $1.5 \mathrm{~h}$, after which time TLC ( silica gel, $\mathrm{CH}_{2} \mathrm{Cl}_{2}$ ) indicated complete consumption of the starting sulfone. The reaction mixture was quenched with $2 \mathrm{~mL}$ of water. The aqueous phase was extracted with $\mathrm{CH}_{2} \mathrm{Cl}_{2}(3 \times 5 \mathrm{~mL})$ and the combined organic solutions were dried over $\mathrm{Na}_{2} \mathrm{SO}_{4}$, filtered, and concentrated in vacuo. The crude product was carried forward without purification.

The crude divinyl carbinol was dissolved in $10 \mathrm{~mL}$ of $\mathrm{CH}_{2} \mathrm{Cl}_{2}$, then $1.72 \mathrm{~g}$ of $\mathrm{MnO}_{2}(24.2$ mmol) was added and the reaction mixture was stirred at RT overnight, after which time TLC (silica gel, $\mathrm{CH}_{2} \mathrm{Cl}_{2}$ ) indicated complete consumption of the carbinol. The reaction mixture was filtered over Celite and the filtrate was concentrated in vacuo. Flash chromatography over silica gel $\left(\mathrm{CH}_{2} \mathrm{Cl}_{2}\right)$ provided $202 \mathrm{mg}$ of 5-methyl-1-phenyl-2-tosylhexa-1,4-dien-3-one (8) (61\% yield over two steps) as a white crystalline solid. Characterization data: IR $\left(\mathrm{CHCl}_{3}\right)$ 3055, 3028, 2917, 2847, 1665, 1609, 1446, 1316, 1148, $1086 \mathrm{~cm}^{-1}$; ${ }^{1} \mathrm{H}$ NMR (400 MHz, $\left.\mathrm{CDCl}_{3}\right) \delta 1.80(\mathrm{~s}, 3 \mathrm{H}), 2.16(\mathrm{~s}, 3 \mathrm{H}), 2.43(\mathrm{~s}, 3 \mathrm{H}), 6.05(\mathrm{~s}$, 1H), 7.29-7.42 (m, 7H), $7.78(\mathrm{~d}, J=8.9 \mathrm{~Hz}, 2 \mathrm{H}), 7.79(\mathrm{~s}, 1 \mathrm{H}) ;{ }^{13} \mathrm{C} \mathrm{NMR}\left(100 \mathrm{MHz}, \mathrm{CDCl}_{3}\right) \delta 21.6$,

\footnotetext{
${ }^{4}$ Eisch, J. J.; Behrooz, M.; Galle, J. E. Tetrahedron Lett. 1984, 25, 4851-4854.

${ }^{5}$ Kostova, K.; Dimitrov, V.; Simova, S.; Hesse, M. Helv. Chim. Acta 1999, 82, 1385-1399.

${ }^{6} \mathrm{Wu}, \mathrm{K}$.; Midland, M. M., Okamura, W. H. J. Org. Chem. 1990, 55, 4381-4392.

${ }^{7}$ Adapted from Enders, D.; Berg, S.; Jandeleit, B. Org. Synth. 2000, 78, 177-188. For characterization data see Hachtel, J.; Gais, H. Eur. J. Org.Chem. 2000, 8, 1457-1466.
} 
21.9, 28.1, 124.9, 128.7, 129.0, 131.1, 132.1, 137.3, 139.5, 143.3, 144.8, 160.9, 190.1; HRMS (DEI): Exact mass calcd for $\left(\mathrm{M}+\mathrm{Na}^{+}\right)$363.10308. Found 363.10318.

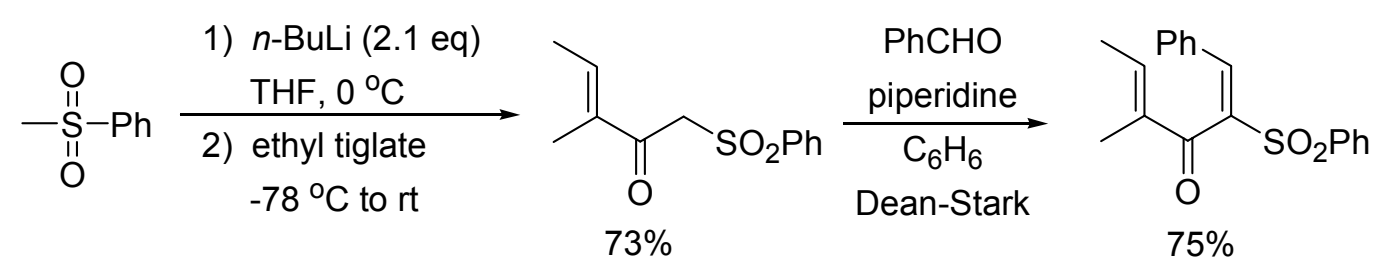

Procedure B. To a solution of $2.34 \mathrm{~g}(15 \mathrm{mmol})$ of methyl phenyl sulfone in $50 \mathrm{~mL}$ of THF cooled to $0{ }^{\circ} \mathrm{C}$ was added dropwise $17.5 \mathrm{~mL}$ of a $2 \mathrm{M}$ solution of $n$ - $\mathrm{BuLi}$ in hexanes $(35 \mathrm{mmol})$. The yellow suspension was stirred at bath temperature for $15 \mathrm{~min}$, cooled to $-78^{\circ} \mathrm{C}$ and then $2.74 \mathrm{~mL}$ of neat ethyl tiglate $(2.30 \mathrm{~g}, 18 \mathrm{mmol})$ was added. The reaction mixture was stirred for $1 \mathrm{~h}$ at $-78{ }^{\circ} \mathrm{C}$, warmed to RT until it became homogeneous, and then quenched with $2 \mathrm{~N}$ aqueous $\mathrm{H}_{2} \mathrm{SO}_{4}$ to $\mathrm{pH} \approx 1-2$. The reaction mixture was partitioned between $50 \mathrm{~mL} \mathrm{H}_{2} \mathrm{O}$ and $100 \mathrm{~mL}$ EtOAc. The organic layer was washed with brine $(50 \mathrm{~mL})$, dried $\left(\mathrm{MgSO}_{4}\right)$, filtered, and concentrated in vacuo. The crude solid was flash chromatographed over silica gel (EtOAc:hexanes, 1:2, then 1:1) to provide $2.6 \mathrm{~g}(73 \%)$ of 1 benzenesulfonyl-3-methylpent-3-en-2-one ${ }^{3}$ as a colorless oil.

A solution of $990 \mathrm{mg}$ ( $4.15 \mathrm{mmol}$ ) of 1-benzenesulfonyl-3-methylpent-3-en-2-one, $0.46 \mathrm{~mL}$ of benzaldehyde ( $484 \mathrm{mg}, 4.57 \mathrm{mmol}$ ), and $21 \mu \mathrm{L}$ of piperidine (18 mg, $0.21 \mathrm{mmol}$ ) in $50 \mathrm{~mL}$ of benzene was refluxed for $15 \mathrm{~h}$ using a Dean-Stark trap. The reaction mixture was then sequentially washed with $25 \mathrm{~mL}$ of sat. $\mathrm{NaHSO}_{3}$ and $25 \mathrm{~mL}$ of sat. $\mathrm{NaHCO}_{3}$, dried $\left(\mathrm{MgSO}_{4}\right)$, filtered, and concentrated in vacuo. The crude solid was recrystallized from $\mathrm{CH}_{2} \mathrm{Cl}_{2} /$ hexanes to give $1.02 \mathrm{~g} \mathrm{(75 \% )}$ of the desired divinyl ketone as a yellow solid. Characterization data: IR (neat) 3065, 2923, 2854, 1725, 1680, 1665, 1611, 1447, 1320, 1310, 1150, $1086 \mathrm{~cm}^{-1} ;{ }^{1} \mathrm{H}$ NMR (400 MHz, $\left.\mathrm{CDCl}_{3}\right) \delta 1.75$ (d, J=7.0 Hz, 3H), 1.84 (s, 3H), $6.82(\mathrm{q}, J=7.0 \mathrm{~Hz}, 1 \mathrm{H}), 7.23-7.27(\mathrm{~m}, 3 \mathrm{H}), 7.54$ (t, $J=7.4 \mathrm{~Hz}, 2 \mathrm{H}), 7.63(\mathrm{t}, J=7.4 \mathrm{~Hz}, 1 \mathrm{H})$, 7.86-7.91 (m, 3H); ${ }^{13} \mathrm{C}$ NMR (100 MHz, $\left.\mathrm{CDCl}_{3}\right) \delta 10.7,15.5,128.6,129.0,129.2,130.1,131.1$, 132.0, 133.7, 138.1, 140.1, 140.2, 141.1, 146.3, 193.4; HRMS (DEI): Exact mass calcd for $\left(\mathrm{M}+\mathrm{Na}^{+}\right)$ 349.08743. Found 349.08765. 


\section{Org. Lett. \\ Supporting Information}

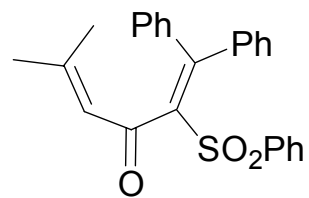

2-Benzenesulfonyl-5-methyl-1,1-diphenylhexa-1,4-dien-3-one.

Following

Procedure A, was obtained in $50 \%$ over two steps. Characterization data: crystalline solid, mp $134-136{ }^{\circ} \mathrm{C}$ (EtOAc:hexane); IR (neat) 3058, 2920, 1668, $1610 \mathrm{~cm}^{-1}$; ${ }^{1} \mathrm{H}$ NMR (400MHz, $\left.\mathrm{CDCl}_{3}\right), 1.73$ (s, 3H), 2.03 (s, 3H), 6.02 (s, 1H), 7.08-7.13 (m, 4H), 7.19-7.38 (m, 8H), 7.48-7.60 (m, 3H); ${ }^{13} \mathrm{C}$ NMR (100 MHz, $\left.\mathrm{CDCl}_{3}\right) \delta$ 21.1, 27.8, 125.8, 127.8, 128.3, 128.4, 128.6, 128.7, 129.2, 129.3, 129.5, 133.1, 137.1, 139.1, 141.9, 144.3, 153.5, 158.8, 189.9; HRMS (DEI): Exact mass calcd for $\left(\mathrm{M}+\mathrm{Na}^{+}\right)$425.11873. Found 425.11810.

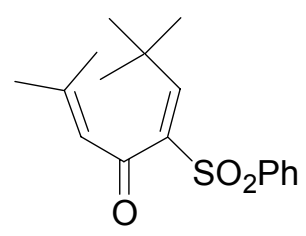

5-Benzenesulfonyl-2,7,7-trimethylocta-1,4-dien-3-one. Following Procedure A, was obtained in $46 \%$ over two steps. Characterization data: IR $\left(\mathrm{CHCl}_{3}\right) 3064$, 2926, 2866, 1669, 1609, 1445, 1316, 1306, 1147, $1085 \mathrm{~cm}^{-1}$; ${ }^{1} \mathrm{H}$ NMR (400 MHz, $\left.\mathrm{CDCl}_{3}\right) \delta 1.10(\mathrm{~s}, 9 \mathrm{H}), 1.90(\mathrm{~s}, 3 \mathrm{H}), 2.09(\mathrm{~s}, 3 \mathrm{H}), 6.25(\mathrm{~s}, 1 \mathrm{H}), 6.82,(\mathrm{~s}, 1 \mathrm{H}), 7.49(\mathrm{t}$, $J=7.5 \mathrm{~Hz}, 2 \mathrm{H}), 7.59(\mathrm{t}, J=7.5 \mathrm{~Hz}, 1 \mathrm{H}), 7.76(\mathrm{~d}, J=7.5 \mathrm{~Hz}, 2 \mathrm{H}) ;{ }^{13} \mathrm{C} \mathrm{NMR}\left(100 \mathrm{MHz}, \mathrm{CDCl}_{3}\right) \delta$; HRMS (DEI): Exact mass calcd for $\left(\mathrm{M}+\mathrm{Na}^{+}\right)$329.11873. Found 329.11914.

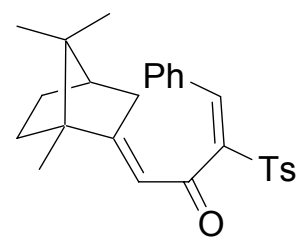

\section{3-Benzene sulfonyl-4-phenyl-1-(1,7,7-trimethyl bicyclo-[2.2.1]-hept-2-ylidene)-} but-3-en-2-one. Following Procedure A, was obtained in 65\% over two steps. Characterization data: crystalline solid, mp $147-148{ }^{\circ} \mathrm{C}$ (EtOAc:hexane); $[\alpha]_{\mathrm{D}}{ }^{23}$ $18\left(\mathrm{CHCl}_{3}, c\right.$ 0.4); IR (neat) 1668, $1601 \mathrm{~cm}^{-1} ;{ }^{1} \mathrm{H} \mathrm{NMR},\left(400 \mathrm{MHz}, \mathrm{CDCl}_{3}\right), \delta 0.58$ $(\mathrm{s}, 3 \mathrm{H}), 0.68(\mathrm{~s}, 3 \mathrm{H}), 1.00-1.14(\mathrm{~m}, 2 \mathrm{H}), 1.60(\mathrm{~m}, 1 \mathrm{H}), 1.75(\mathrm{~m}, 1 \mathrm{H}), 1.84(\mathrm{t}, J=14.0 \mathrm{~Hz}, 1 \mathrm{H}), 2.40(\mathrm{~d}$, $J=19.0 \mathrm{~Hz}, 1 \mathrm{H}), 2.43$ (s, 3H), 2.82 (d, $J=19.0 \mathrm{~Hz}, 1 \mathrm{H}), 5.85(\mathrm{~s}, 1 \mathrm{H}), 7.28-7.39$ (m, 7H), 7.81 (d, $J=$ $8 \mathrm{~Hz}, 2 \mathrm{H}), 7.88(\mathrm{~s}, 1 \mathrm{H}) ;{ }^{13} \mathrm{C} \mathrm{NMR}\left(100 \mathrm{MHz}, \mathrm{CDCl}_{3}\right) \delta 12.3,19.0,19.8,21.9,27.2,33.8,39.7,44.6$, 48.6, 54.6, 117.3, 128.8, 128.9, 129.9, 130.3, 131.0, 132.5, 137.7, 140.0, 143.7, 144.7, 179.3, 189.7; HRMS (DEI): Exact mass calcd for $\left(\mathrm{M}+\mathrm{Na}^{+}\right)$457.18133. Found 457.18149.

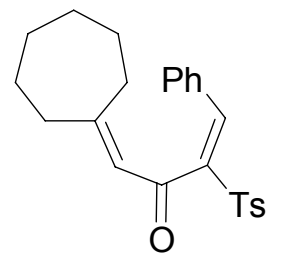

3-Benzenesulfonyl-1-cycloheptylidene-4-phenylbut-3-en-2-one.

Following

Procedure A, was obtained in $51 \%$ over two steps. Characterization data: crystalline solid, mp $135-136{ }^{\circ} \mathrm{C}$ (EtOAc:hexane); IR (neat) $1659,1595 \mathrm{~cm}^{-1} ;{ }^{1} \mathrm{H}$

NMR (400 MHz, $\left.\mathrm{CDCl}_{3}\right) \delta 1.47(\mathrm{~m}, 6 \mathrm{H}), 1.66(\mathrm{~m}, 2 \mathrm{H}), 2.21(\mathrm{~m}, 2 \mathrm{H}), 2.44(\mathrm{~s}, 3 \mathrm{H})$, $2.88(\mathrm{dd}, J=5.6 \mathrm{~Hz}, 2 \mathrm{H}), 6.01(\mathrm{~s}, 1 \mathrm{H}), 7.32-7.40(\mathrm{~m}, 7 \mathrm{H}), 7.79(\mathrm{~s}, 1 \mathrm{H}), 7.82(\mathrm{~d}, J=4.0 \mathrm{~Hz}, 2 \mathrm{H}) ;{ }^{13} \mathrm{C}$

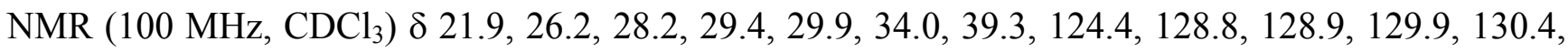




\section{Org. Lett. \\ Supporting Information}

131.1, 132.3, 137.4, 139.4, 143.7, 144.7, 171.9, 189.7; HRMS (DEI): Exact mass calcd for $\left(\mathrm{M}+\mathrm{Na}^{+}\right)$ 417.15003. Found 417.15033.

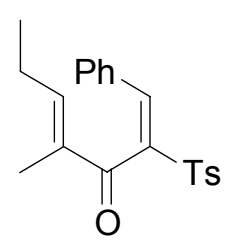

2-Benzenesulfonyl-4-methyl-1-phenylhepta-1,4-dien-3-one. Following Procedure A, was obtained in $79 \%$ over two steps. Characterization data: crystalline solid, mp 112-114 ${ }^{\circ} \mathrm{C}$ (EtOAc:hexane); IR 2967, 1649, $1633 \mathrm{~cm}^{-1} ;{ }^{1} \mathrm{H} \mathrm{NMR}\left(400 \mathrm{MHz}, \mathrm{CDCl}_{3}\right) \delta$ $0.72(\mathrm{t}, J=7.6 \mathrm{~Hz}, 3 \mathrm{H}), 1.78(\mathrm{~s}, 3 \mathrm{H}), 2.07$ (q, $J=7.3 \mathrm{~Hz}, 2 \mathrm{H}), 2.42(\mathrm{~s}, 3 \mathrm{H}), 6.61$ (dt, $J=$ 6.8, 0.8 Hz, 1H), 7.21-7.39 (m, 7H), $7.77(\mathrm{~d}, J=8.4 \mathrm{~Hz}, 2 \mathrm{H}), 7.90(\mathrm{~s}, 1 \mathrm{H}) ;{ }^{13} \mathrm{C} \mathrm{NMR}(100 \mathrm{MHz}$, $\left.\mathrm{CDCl}_{3}\right) \delta 10.9,12.4,21.8,22.8,128.7,128.7,130.0,130.1,130.9,132.4,136.3,137.2,140.56,141.0$, 144.8, 152.6, 193.8; HRMS (DEI): Exact mass calcd for $\left(\mathrm{M}+\mathrm{Na}^{+}\right)$377.11873. Found 377.11768.

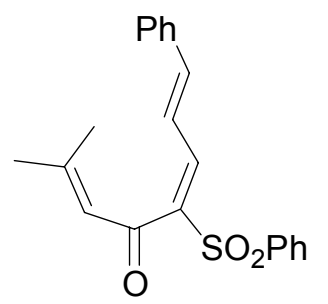

5-Benzenesulfonyl-2-methyl-8-phenylocta-2,5,7-trien-4-one (20). Beginning with 1-benzenesulfonyl-4-methylpent-3-en-2-one ${ }^{3}$ and following Procedure B, the Knoevenagel reaction with 3-phenylpropenal provided $\mathbf{2 0}$ as a colorless solid in $71 \%$ yield. Characterization data: crystalline solid, mp $119-120{ }^{\circ} \mathrm{C}$ (EtOAc:hexane); IR (neat) 2955, 1722, 1658, 1608, 1579, $1314 \mathrm{~cm}^{-1} ;{ }^{1} \mathrm{H}$ NMR $\left(400 \mathrm{MHz}, \mathrm{CDCl}_{3}\right) \delta 1.97(\mathrm{~s}, 3 \mathrm{H}), 2.15(\mathrm{~s}, 3 \mathrm{H}), 6.42(\mathrm{~s}, 1 \mathrm{H}), 7.15(\mathrm{~d}, J=9.0 \mathrm{~Hz}, 1 \mathrm{H}), 7.17(\mathrm{~s}, 1 \mathrm{H})$, 7.38-7.41 (m, 3H), 7.48-7.62 (m, 5H), $7.21(\mathrm{dd}, J=9.0,2.1 \mathrm{~Hz}, 1 \mathrm{H}), 7.91(\mathrm{~d}, J=7.4 \mathrm{~Hz}, 2 \mathrm{H}) ;{ }^{13} \mathrm{C}$ NMR $\left(100 \mathrm{MHz}, \mathrm{CDCl}_{3}\right) \delta 21.7,28.3,122.3,125.0,128.2,128.3,129.2,129.2,130.5,133.5,135.5$, 141.0, 141.5, 143.7, 146.8, 160.2, 187.7; HRMS (DEI): Exact mass calcd for $\left(\mathrm{M}+\mathrm{Na}^{+}\right) 375.10308$. Found 375.10312.

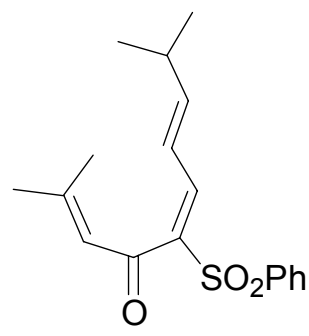

5-Benzenesulfonyl-2,9-dimethyldeca-2,5,7-trien-4-one (22). Beginning with 1benzenesulfonyl-4-methylpent-3-en-2-one ${ }^{3}$ and following Procedure $\mathbf{B}$, the Knoevenagel reaction with 4-methylpent-2-enal provided 22 as a colorless oil in $34 \%$ over two steps. Characterization data: IR (neat) 2963, 2931, 1662, $1609 \mathrm{~cm}^{-}$

${ }^{1} ;{ }^{1} \mathrm{H}$ NMR (400 MHz, $\left.\mathrm{CDCl}_{3}\right) \delta 1.05(\mathrm{~d}, J=6.8 \mathrm{~Hz}, 6 \mathrm{H}), 1.92$ (s, 3H), 2.09 (s, $3 \mathrm{H}), 2.47(\mathrm{~m}, 1 \mathrm{H}), 6.31-6.39(\mathrm{~m}, 2 \mathrm{H}), 7.36(\mathrm{~s}, 1 \mathrm{H}), 7.47-7.60(\mathrm{~m}, 4 \mathrm{H}), 7.86(\mathrm{~d}, J=8.0 \mathrm{~Hz}, 2 \mathrm{H}) ;{ }^{13} \mathrm{C}$ NMR $\left(100 \mathrm{MHz}, \mathrm{CDCl}_{3}\right) \delta 21.6,21.7,28.2,32.3,122.1,125,0,128.3,128.6,129.1,133.4,140.3$, 141.1, 144.1, 158.5, 159.9, 187.9; HRMS (DEI): Exact mass calcd for $\left(\mathrm{M}+\mathrm{Na}^{+}\right)$341.11873. Found 341.11746. 
Org. Lett.

Supporting Information

\section{$\underline{\text { General Procedure for Divinyl Ketone/Cyclohexenone Cyclizations }}$}
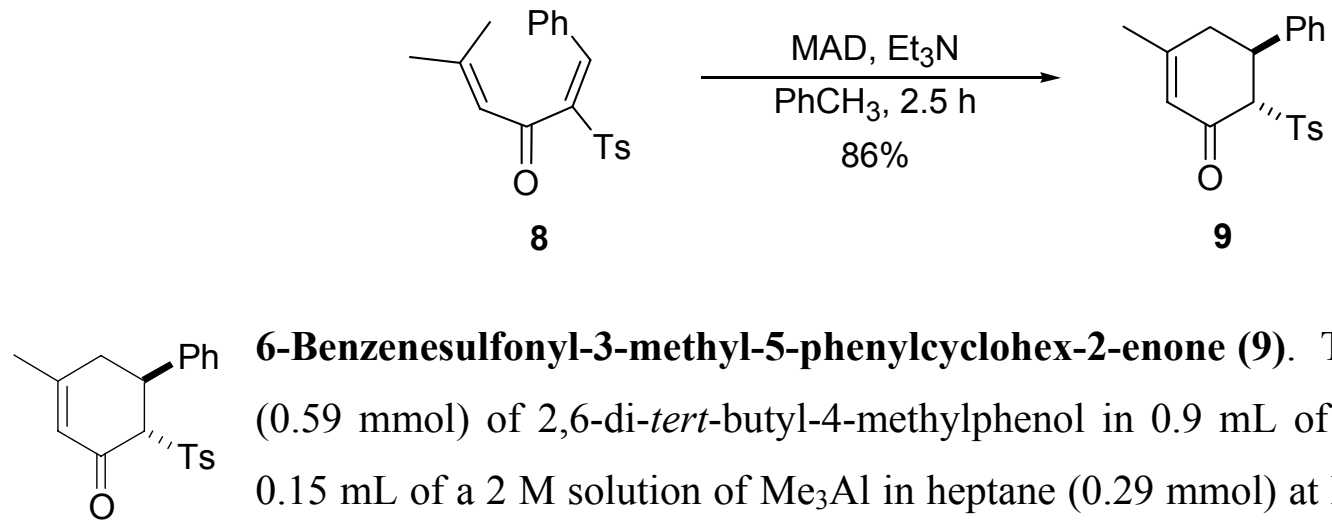

6-Benzenesulfonyl-3-methyl-5-phenylcyclohex-2-enone (9). To a solution of $129 \mathrm{mg}$ (0.59 mmol) of 2,6-di-tert-butyl-4-methylphenol in $0.9 \mathrm{~mL}$ of dry $\mathrm{PhCH}_{3}$ was added $0.15 \mathrm{~mL}$ of a $2 \mathrm{M}$ solution of $\mathrm{Me}_{3} \mathrm{Al}$ in heptane $(0.29 \mathrm{mmol})$ at RT. After stirring for 1 $\mathrm{h}$, the solution was cooled to $0{ }^{\circ} \mathrm{C}$ and $22.5 \mu \mathrm{L}$ of $\mathrm{Et}_{3} \mathrm{~N}$ (16 mg, $\left.0.16 \mathrm{mmol}\right)$ was added followed immediately by a solution of divinyl ketone $8 \mathrm{in} 2 \mathrm{~mL}$ of dry $\mathrm{PhCH}_{3}$. The reaction mixture was stirred at $0{ }^{\circ} \mathrm{C}$ for $15 \mathrm{~min}$ and then was warmed to RT over $2.5 \mathrm{~h}$ after which time it was quenched with $3 \mathrm{~mL}$ of sat. aqueous $\mathrm{NH}_{4} \mathrm{Cl}$. After diluting with $5 \mathrm{~mL} \mathrm{CH}_{2} \mathrm{Cl}_{2}$, the aqueous phase was separated and extracted with $\mathrm{CH}_{2} \mathrm{Cl}_{2}(3 \times 10 \mathrm{~mL})$. The combined organic solutions were dried $\left(\mathrm{Na}_{2} \mathrm{SO}_{4}\right)$, filtered, and concentrated in vacuo. Flash chromatography over silica gel $\left(\mathrm{CH}_{2} \mathrm{Cl}_{2}\right)$ provided $43 \mathrm{mg}(86 \%)$ of cyclohexenone 9 as a colorless oil. Characterization data: IR $\left(\mathrm{CHCl}_{3}\right)$ 3030, 2917, 2848, 1661, 1633, 1495, 1451, 1317, 1145, $1084 \mathrm{~cm}^{-1} ;{ }^{1} \mathrm{H}$ NMR (400 MHz, $\left.\mathrm{CDCl}_{3}\right) \delta 2.07$ (s, 3H), 2.45 (s, 3H), $2.56(\mathrm{~d}, J$ $=19.4 \mathrm{~Hz}, 1 \mathrm{H}), 3.44(\mathrm{dd}, J=19.3,6.8 \mathrm{~Hz}, 1 \mathrm{H}), 3.95(\mathrm{~s}, 1 \mathrm{H}), 4.44(\mathrm{~d}, J=6.4 \mathrm{~Hz}, 1 \mathrm{H}), 6.10(\mathrm{~s}, 1 \mathrm{H})$, 7.06-7.11 (m, 2H), 7.18-7.28 (m, 3H), 7.35 (d, $J=8.1 \mathrm{~Hz}, 2 \mathrm{H}), 7.71(\mathrm{~d}, J=8.4 \mathrm{~Hz}, 2 \mathrm{H})$; ${ }^{13} \mathrm{C}$ NMR $\left(100 \mathrm{MHz} \mathrm{CDCl}_{3}\right) \delta 21.9,25.0,29.9,34.9,38.2,73.9,109.6,126.8,127.3,127.5,129.1,129.2,130.0$, 135.3, 142.2, 145.5, 162.9, 188.2; HRMS (DEI): Exact mass calcd for $\left(\mathrm{M}+\mathrm{Na}^{+}\right)$363.10308. Found 363.10196.

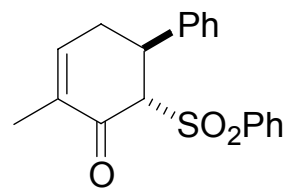

6-Benzenesulfonyl-2-methyl-5-phenylcyclohex-2-enone (10). Once all of the reagents were combined, the reaction mixture was stirred for $1 \mathrm{~h}$ at $60{ }^{\circ} \mathrm{C}$. After the usual workup, the crude material was purified by flash chromatography (silica gel, EtOAc:hexanes, 1:2) to provide 10 as a colorless oil (76\%). Characterization data: IR (neat) 3062, 3029, 2923, 1724, 1667, 1496, 1448, 1309, 1148, $1083 \mathrm{~cm}^{-1} ;{ }^{1} \mathrm{H}$ NMR $\left(400 \mathrm{MHz}, \mathrm{CDCl}_{3}\right) \delta 1.85(\mathrm{t}, J=$ $1.4 \mathrm{~Hz}, 3 \mathrm{H}), 2.68$ (dd, $J=19.8,5.8 \mathrm{~Hz}, 1 \mathrm{H}), 3.45$ (dddd, $J=19.9,9.3,5.4,2.7 \mathrm{~Hz}, 1 \mathrm{H}), 4.10(\mathrm{~s}, 1 \mathrm{H})$, $4.43(\mathrm{~d}, J=6.6 \mathrm{~Hz}, 1 \mathrm{H}), 6.86-6.91(\mathrm{~m}, 1 \mathrm{H}), 7.09-7.13(\mathrm{~m}, 2 \mathrm{H}), 7.19-7.29(\mathrm{~m}, 3 \mathrm{H}), 7.57$ (t, $J=7.4 \mathrm{~Hz}$, 2H), $7.68(\mathrm{t}, J=7.4 \mathrm{~Hz}, 1 \mathrm{H}), 7.80-7.84(\mathrm{~m}, 2 \mathrm{H}) ;{ }^{13} \mathrm{C} \mathrm{NMR}\left(100 \mathrm{MHz}, \mathrm{CDCl}_{3}\right) \delta 16.5,29.9,38.8,75.0$, 
127.3, 127.4, 129.0, 129.1, 129.3, 134.3, 136.1, 128.5, 142.1, 145.3, 188.9; HRMS (DEI): Exact mass calcd for $\left(\mathrm{M}+\mathrm{Na}^{+}\right)$349.08743. Found 349.08743.

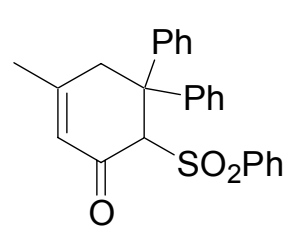

6-Benzenesulfonyl-3-methyl-5,5-diphenylcyclohex-2-enone (11). Once all of the reagents were combined ( $N$-methylpyrrolidine was used instead of triethylamine), the reaction mixture was stirred for $4 \mathrm{~h}$ at $60{ }^{\circ} \mathrm{C}$. After the usual workup, the crude was purified by flash chromatography (silica gel, EtOAc:hexanes, 1:2) to provide 11 as a white crystalline solid (74\%). Characterization data: mp 220-221 ${ }^{\circ} \mathrm{C}$ (EtOAc:hexane); IR (neat) 2851, 1661, $1313 \mathrm{~cm}^{-1} ;{ }^{1} \mathrm{H} \mathrm{NMR}\left(400 \mathrm{MHz}, \mathrm{CDCl}_{3}\right) \delta 2.03(\mathrm{~s}, 3 \mathrm{H}), 3.18(\mathrm{~d}, J=18.0 \mathrm{~Hz}, 1 \mathrm{H})$, $4.53(\mathrm{~d}, J=18.0 \mathrm{~Hz}, 1 \mathrm{H}), 5.00(\mathrm{~s}, 1 \mathrm{H}), 6.02(\mathrm{~s}, 1 \mathrm{H}), 7.07-7.19(\mathrm{~m}, 10 \mathrm{H}), 7.44-7.53(\mathrm{~m}, 5 \mathrm{H}) ;{ }^{13} \mathrm{C} \mathrm{NMR}$ $\left(100 \mathrm{MHz}, \mathrm{CDCl}_{3}\right) \delta 25.5,39.2,51.0,78.3,126.1,126.4,126.9,127.1,127.4,128.2,128.3,128.5$, 128.8, 129.0, 133.5, 140.9, 143.2, 146.4, 162.7, 189.6; HRMS (DEI): Exact mass calcd for $\left(\mathrm{M}+\mathrm{Na}^{+}\right)$ 425.11873. Found 425.11923.

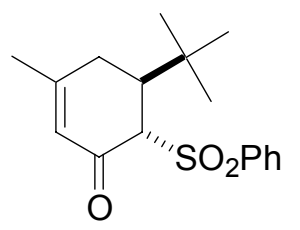

6-Benzenesulfonyl-5-tert-butyl-3-methylcyclohex-2-enone (12). Once all of the reagents were combined, the reaction stirred for $12 \mathrm{~h}$ at RT. After the usual workup, the crude was purified by flash chromatography (silica gel, EtOAc:hexanes, 1:3) to provide 12 as a colorless oil (91\%). Characterization data: IR $\left(\mathrm{CHCl}_{3}\right)$ 3058, 2928, 2865, 1644, 1316, 1307, 1250, 1144, $1083 \mathrm{~cm}^{-1} ;{ }^{1} \mathrm{H}$ NMR (400 MHz, $\left.\mathrm{CDCl}_{3}\right)$ $\delta 0.86(\mathrm{~s}, 9 \mathrm{H}), 2.01(\mathrm{~s}, 3 \mathrm{H}), 2.41(\mathrm{~d}, J=19.7 \mathrm{~Hz}, 1 \mathrm{H}), 2.84(\mathrm{~d}, J=7.9 \mathrm{~Hz}, 1 \mathrm{H}), 2.96(\mathrm{dd}, J=19.8,7.6$ $\mathrm{Hz}, 1 \mathrm{H}), 3.94$ (s, 1H), 5.97 (s, 1H), 7.55 (t, $J=7.6 \mathrm{~Hz}, 2 \mathrm{H}), 7.66$ (t, $J=7.4 \mathrm{~Hz}, 1 \mathrm{H}), 7.80$ (d, $J=7.1$ $\mathrm{Hz}, 1 \mathrm{H}) ;{ }^{13} \mathrm{C} \mathrm{NMR}\left(100 \mathrm{MHz}, \mathrm{CDCl}_{3}\right) \delta 24.9,28.2,30.0,34.9,42.4,71.1,126.0,129.1,129.2,134.2$, 138.3, 164.3, 189.1; HRMS (DEI): Exact mass calcd for $\left(\mathrm{M}+\mathrm{Na}^{+}\right)$329.11873. Found 329.11764.

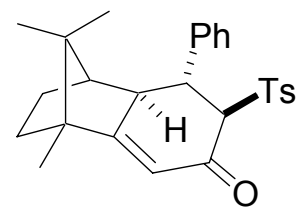

4,9,9-Trimethyl-8-phenyl-7-(toluene-4-sulfonyl)-1,3,4,7,8,8a-hexahydro-2H1,4-methanonaphthalen-6-one (14). Once all of the reagents were combined $(N$ methylpyrrolidine was used instead of triethylamine), the reaction was stirred for 4 h at $0{ }^{\circ} \mathrm{C}$. After the usual workup, the crude material was purified by flash chromatography (silica gel, EtOAc:hexanes, 1:4) to provide 14 as a colorless oil (91\%). Characterization data: $[\alpha]_{\mathrm{D}}{ }^{23}-31\left(\mathrm{CHCl}_{3}\right.$, c 0.3); IR (neat) 2936, 2927, 1665, $1659 \mathrm{~cm}^{-1} ;{ }^{1} \mathrm{H}$ NMR (400 MHz, $\left.\mathrm{CDCl}_{3}\right) \delta 0.93(\mathrm{~s}, 3 \mathrm{H}), 1.02(\mathrm{~m}$, $1 \mathrm{H}), 1.04(\mathrm{~s}, 3 \mathrm{H}), 1.10(\mathrm{~s}, 3 \mathrm{H}), 1.45-1.71(\mathrm{~m}, 4 \mathrm{H}), 2.34(\mathrm{dd}, J=12.0,2.2 \mathrm{~Hz}, 1 \mathrm{H}), 2.41(\mathrm{~s}, 3 \mathrm{H}), 3.76$ 
(dd, $J=12.0,10.0 \mathrm{~Hz}, 1 \mathrm{H}), 4.28(\mathrm{~d}, J=9.6 \mathrm{~Hz}, 1 \mathrm{H}), 5.95(\mathrm{~d}, J=1.2 \mathrm{~Hz}, 1 \mathrm{H}), 7.22-7.36(\mathrm{~m}, 7 \mathrm{H}), 7.56$ $(\mathrm{d}, J=8.1 \mathrm{~Hz}, 2 \mathrm{H}) ;{ }^{13} \mathrm{C} \mathrm{NMR}\left(100 \mathrm{MHz}, \mathrm{CDCl}_{3}\right) \delta 11.6,20.8,21.6,21.8,30.2,32.0,45.3,46.1,49.1$, 52.1, 53.3, 76.3, 117.4, 127.2, 128.2, 128.9, 129.3, 129.6, 136.6, 142.5, 144.8, 179.2, 189.3; HRMS (DEI): Exact mass calcd for $\left(\mathrm{M}+\mathrm{Na}^{+}\right)$457.18133. Found 457.18024.

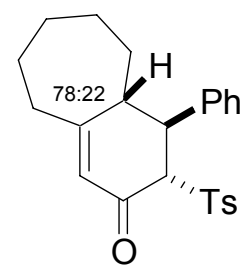

\section{4-Phenyl-3-(toluene-4-sulfonyl)-3,4,4a,5,6,7,8,9-octahydrobenzocyclohepten-2-one}

(15). Once all of the reagents were combined, the reaction mixture was stirred for $2 \mathrm{~h}$ at RT. After the usual workup, the crude material was purified by flash chromatography (silica gel, EtOAc:hexanes, 1:4) to provide $\mathbf{1 5}$ as a white crystalline solid (77\%). Characterization data for the major isomer: mp $153-154{ }^{\circ} \mathrm{C}$ (EtOAc:hexane); IR (neat) 3029, 1737, $1453 \mathrm{~cm}^{-1} ;{ }^{1} \mathrm{H}$ NMR (400 MHz, $\left.\mathrm{CDCl}_{3}\right) \delta 1.50(\mathrm{~m}, 2 \mathrm{H}), 1.74(\mathrm{~m}, 3 \mathrm{H}), 1.84(\mathrm{~m}, 1 \mathrm{H}), 2.07$ (m, 2H), $2.54(\mathrm{~m}, 2 \mathrm{H}), 2.77(\mathrm{~m}, 1 \mathrm{H}), 4.09(\mathrm{~m}, 2 \mathrm{H}), 6.13(\mathrm{~s}, 1 \mathrm{H}), 7.15$ (d, $J=7.8 \mathrm{~Hz}, 2 \mathrm{H}), 7.15-7.32$ $(\mathrm{m}, 5 \mathrm{H}), 7.67(\mathrm{~d}, J=8.0 \mathrm{~Hz}, 2 \mathrm{H}) ;{ }^{13} \mathrm{C} \mathrm{NMR}\left(100 \mathrm{MHz}, \mathrm{CDCl}_{3}\right) \delta 21.9,26.5,27.7,28.4,34.7,37.6$, 45.4, 45.8, 74.0, 126.7, 127.4, 127.5, 129.2, 129.8, 136.8, 144.4, 145.0, 171.2, 188.4; HRMS (DEI): Exact mass calcd for $\left(\mathrm{M}+\mathrm{Na}^{+}\right)$417.15003. Found 417.14917.

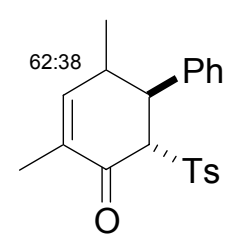

2,4-Dimethyl-5-phenyl-6-(toluene-4-sulfonyl)-cyclohexen-2-one (16). Once all of the reagents were combined, the reaction mixture was stirred for $2 \mathrm{~h}$ at $60{ }^{\circ} \mathrm{C}$. After the usual workup, the crude material was purified by flash chromatography (silica gel, EtOAc:hexanes, 1:4) to provide $\mathbf{1 6}$ as a white crystalline solid (92\%). The two diastereomers are inseparable by flash column chromatography or crystallization. Thus, the two isomers cocrystallized in a 66:34 ratio from EtOAc/hexane, as confirmed by the X-ray crystallography.

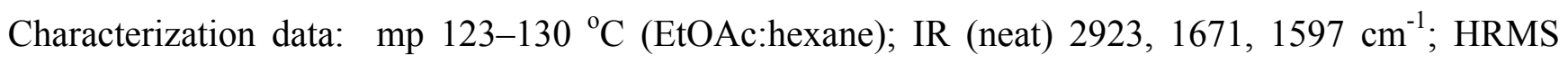
(DEI): Exact mass calcd for $\left(\mathrm{M}+\mathrm{Na}^{+}\right)$377.18173. Found 377.11935.

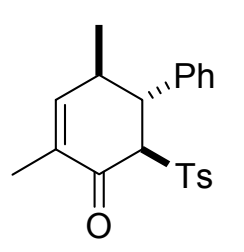

NMR data for the major isomer, 2,4r-Dimethyl-5t-phenyl-6c-(toluene-4-sulfonyl)cyclohex-2-enone: ${ }^{1} \mathrm{H}$ NMR $\left(400 \mathrm{MHz}, \mathrm{CDCl}_{3}\right) \delta 1.37$ (d, $\left.J=7.5 \mathrm{~Hz}, 3 \mathrm{H}\right), 1.90(\mathrm{~d}, J=$ $1.3 \mathrm{~Hz}, 3 \mathrm{H}), 2.43(\mathrm{~s}, 3 \mathrm{H}), 2.76(\mathrm{~m}, 1 \mathrm{H}), 3.86(\mathrm{t}, J=4.6 \mathrm{~Hz}, 1 \mathrm{H}), 4.13(\mathrm{~d}, J=4.8 \mathrm{~Hz}$, $1 \mathrm{H}), 6.69(\mathrm{~d}, J=2.2 \mathrm{~Hz}, 1 \mathrm{H}), 7.16(\mathrm{~d}, J=7.9 \mathrm{~Hz}, 2 \mathrm{H}), 7.22-7.38(\mathrm{~m}, 5 \mathrm{H}), 7.62(\mathrm{~d}, J=$

$7.9 \mathrm{~Hz}, 2 \mathrm{H}) ;{ }^{13} \mathrm{C} \mathrm{NMR}\left(100 \mathrm{MHz}, \mathrm{CDCl}_{3}\right) \delta 16.56,20.6,21.9,36.5,45.6,74.7,127.4,127.4,129.1$, $129.1,129.8,135.4,136.2,143.8,145.0,150.0,189.6$. 


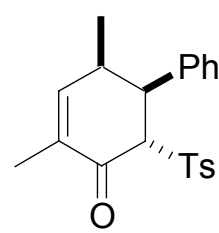

NMR data for the minor isomer, 2,4r-Dimethyl-5c-phenyl-6t-(toluene-4-sulfonyl)cyclohex-2-enone: ${ }^{1} \mathrm{H} \mathrm{NMR},\left(400 \mathrm{MHz}, \mathrm{CDCl}_{3}\right) \delta 1.15(\mathrm{~d}, J=7.2 \mathrm{~Hz}, 3 \mathrm{H}), 1.85$ (s, $3 \mathrm{H}), 2.36(\mathrm{~s}, 3 \mathrm{H}), 3.50(\mathrm{dd}, J=5.0,4.6 \mathrm{~Hz}, 1 \mathrm{H}), 3.92(\mathrm{~m}, 1 \mathrm{H}), 4.26(\mathrm{~d}, J=4.6 \mathrm{~Hz}, 1 \mathrm{H})$, $6.83(\mathrm{~d}, J=0.8 \mathrm{~Hz}, 1 \mathrm{H}), 7.12(\mathrm{~d}, J=8.8 \mathrm{~Hz}, 2 \mathrm{H}), 7.22-7.38(\mathrm{~m}, 7 \mathrm{H}) ;{ }^{13} \mathrm{C}$ NMR $(100$ $\left.\mathrm{MHz}, \mathrm{CDCl}_{3}\right) \delta 16.5,19.8,21.8,32.6,50.9,76.7,127.7,128.4,128.7,129.2,129.5,133.8,137.0$, 137.4, 144.7, 153.1, 191.2.

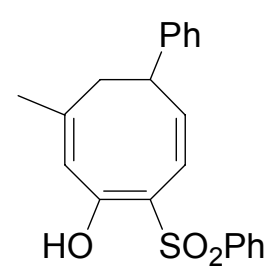

2-Benzenesulfonyl-7-methyl-5-phenylcycloocta-1,3,7-trienol (21). Once all of the reagents were combined, the reaction mixture was stirred for $5 \mathrm{~min}$ at RT. After the usual workup, the crude material was purified by flash chromatography (silica gel, EtOAc:hexanes, 1:4) to provide 21 as a colorless solid (99\%). Characterization data: mp 131-134 ${ }^{\circ} \mathrm{C}$ (EtOAc:hexane); IR (neat) 2923, 2852, $1305 \mathrm{~cm}^{-1} ;{ }^{1} \mathrm{H}$ NMR (400 MHz, $\left.\mathrm{CDCl}_{3}\right) \delta 1.91$ (s, 3H), $2.10(\mathrm{~m}, 1 \mathrm{H}), 2.71(\mathrm{dd}, J=13.6,13.6 \mathrm{~Hz}, 1 \mathrm{H}), 3.49(\mathrm{~m}, 1 \mathrm{H}), 5.72(\mathrm{dd}, J=12.0,6.0 \mathrm{~Hz}, 1 \mathrm{H})$, $5.74(\mathrm{~s}, 1 \mathrm{H}), 6.51(\mathrm{~d}, J=6.4 \mathrm{~Hz}, 2 \mathrm{H}), 7.06-7.12(\mathrm{~m}, 3 \mathrm{H}), 7.57(\mathrm{t}, J=7.6 \mathrm{~Hz}, 2 \mathrm{H}), 7.59-7.71(\mathrm{~m}, 1 \mathrm{H})$, $7.96(\mathrm{~d}, J=7.2 \mathrm{~Hz}, 2 \mathrm{H}) ;{ }^{13} \mathrm{C} \mathrm{NMR}\left(100 \mathrm{MHz}, \mathrm{CDCl}_{3}\right) \delta 25.5,40.5,42.8,107.2,119.0,120.9,126.8$, 127.3, 127.5, 128.6, 129.5, 133.6, 137.8, 141.5, 143.1, 148.8, 160.6; HRMS (DEI): Exact mass calcd for $\left(\mathrm{M}+\mathrm{Na}^{+}\right)$375.10308. Found 375.10239.

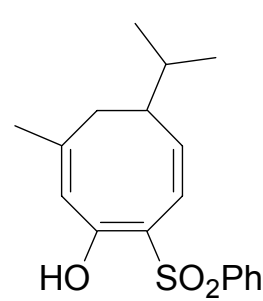

2-Benzene sulfonyl-5-isopropyl-7-methyl-cycloocta-1,3,7-trienol (23). Once all of the reagents were combined, the reaction mixture was stirred for $15 \mathrm{~min}$ at RT. After the usual workup, the crude product was purified by flash chromatography (silica gel, EtOAc:hexanes, 1:4) to provide 23 as a colorless oil (60\%). Characterization data for the enol tautomer: IR (neat) 3198, 2960, 1590, $1273 \mathrm{~cm}^{-1} ;{ }^{1} \mathrm{H}$ NMR (400 MHz, $\left.\mathrm{CDCl}_{3}\right) \delta 0.43(\mathrm{~d}, J=6.8 \mathrm{~Hz}, 3 \mathrm{H}), 0.50(\mathrm{~d}, J=6.8 \mathrm{~Hz}, 3 \mathrm{H}), 1.28(\mathrm{~m}, 1 \mathrm{H}), 2.12(\mathrm{~m}, 3 \mathrm{H}), 5.63(\mathrm{dd}, J=$ 12.0, $6.8 \mathrm{~Hz}, 1 \mathrm{H}), 5.76(\mathrm{~s}, 1 \mathrm{H}), 6.02(\mathrm{~d}, J=11.0 \mathrm{~Hz}, 1 \mathrm{H}), 7.50-7.64(\mathrm{~m}, 3 \mathrm{H}), 7.99$ (d, $J=8 \mathrm{~Hz}, 2 \mathrm{H})$, $10.80(\mathrm{~s}, 1 \mathrm{H}) ;{ }^{13} \mathrm{C} \mathrm{NMR}\left(100 \mathrm{MHz}, \mathrm{CDCl}_{3}\right) \delta$ 19.4, 19.6, 28.0, 32.4, 36.6, 42.1, 107.2, 120.0, 120.1, 127.5, 127.3, 133.4, 140.6, 141.3, 148.8, 160.2; HRMS (DEI): Exact mass calcd for $\left(\mathrm{M}+\mathrm{Na}^{+}\right)$ 341.11873. Found 341.11719. 


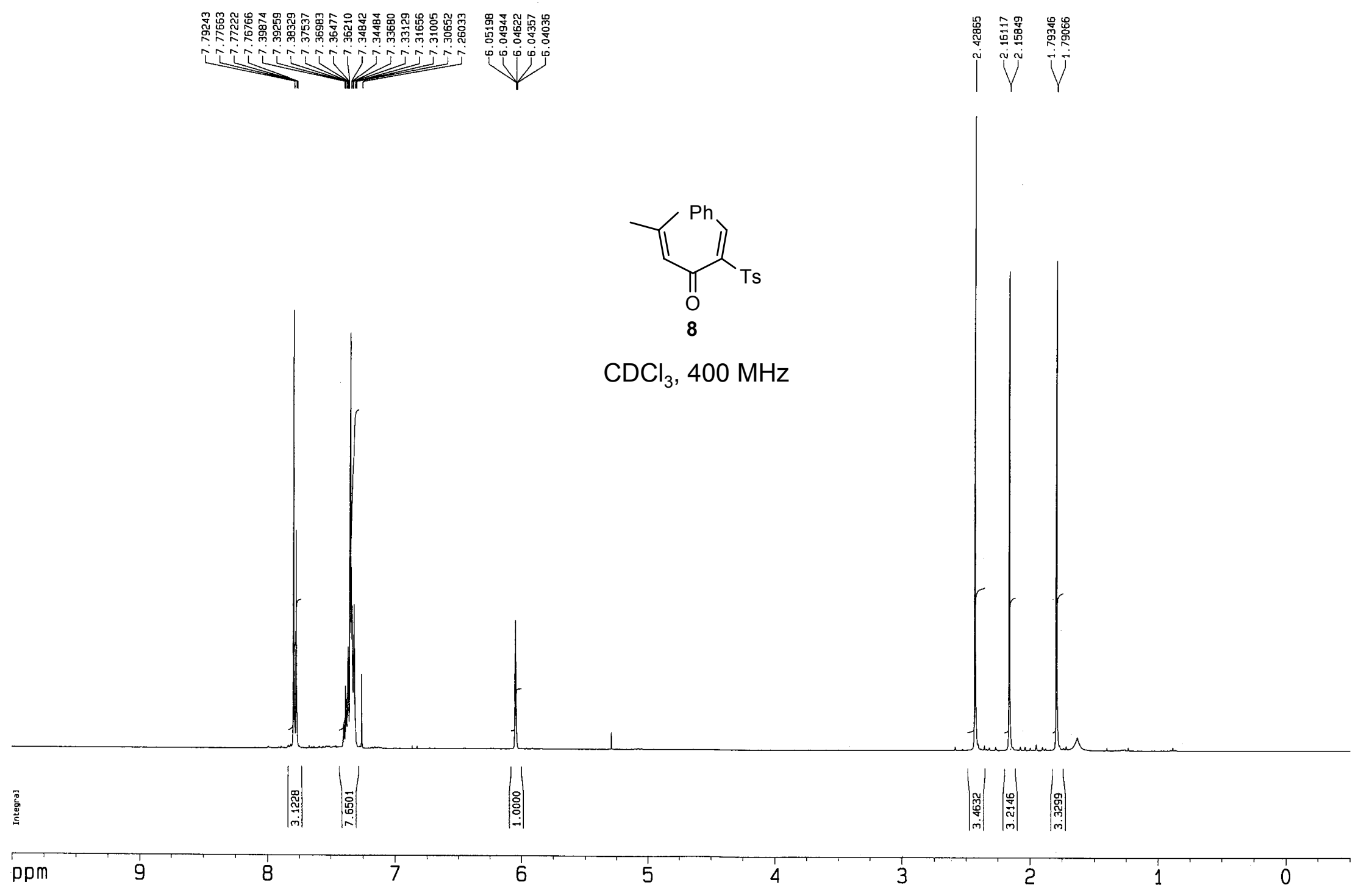




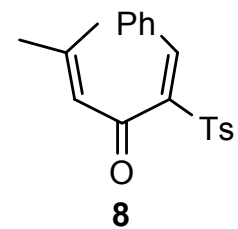

$\mathrm{CDCl}_{3}, 100 \mathrm{MHz}$ 


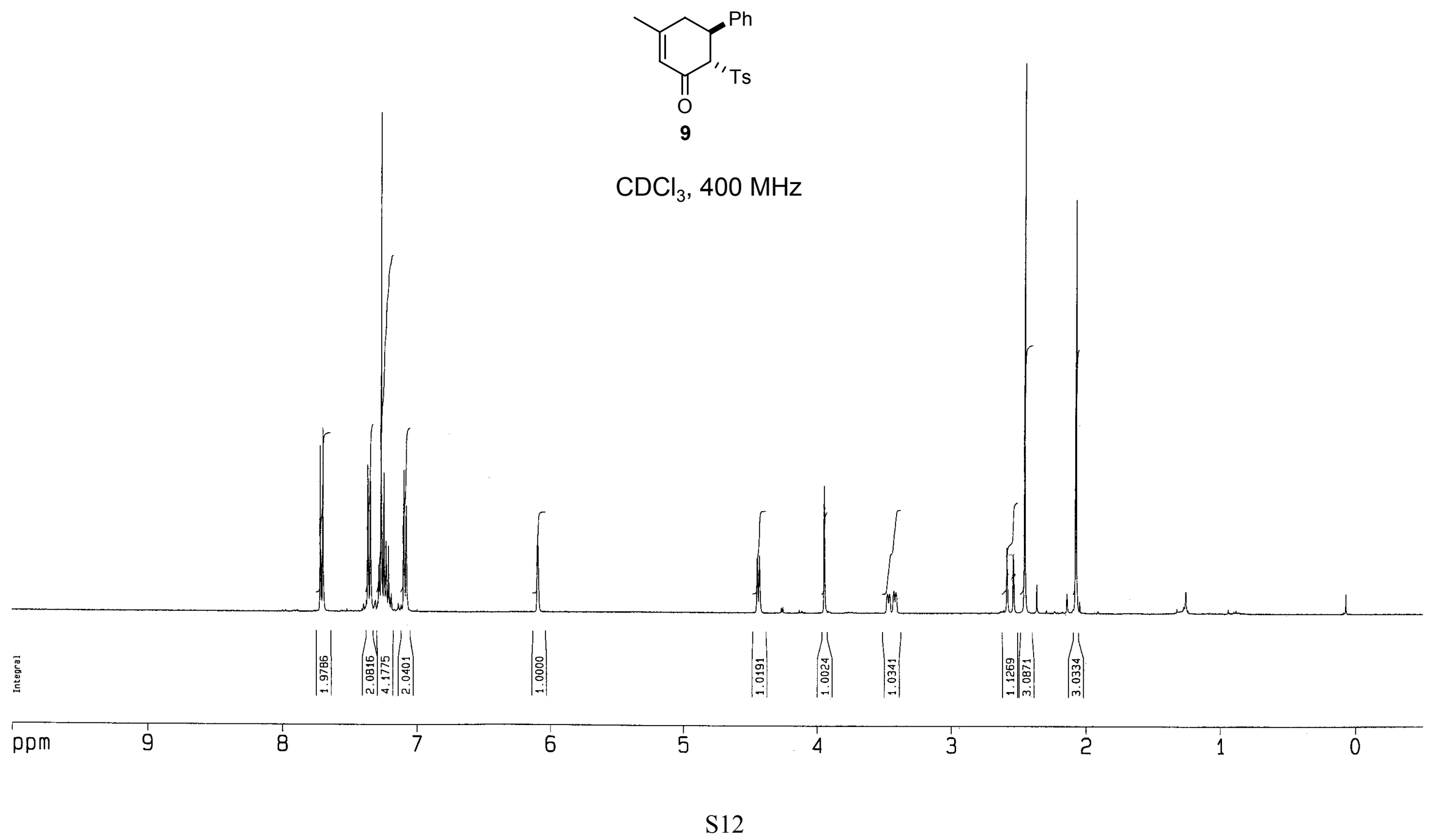




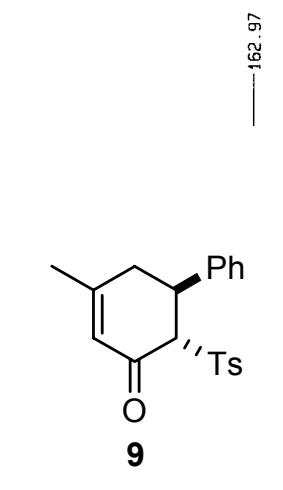

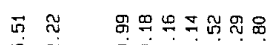

ป N

îj

$\mathrm{CDCl}_{3}, 100 \mathrm{MHz}$

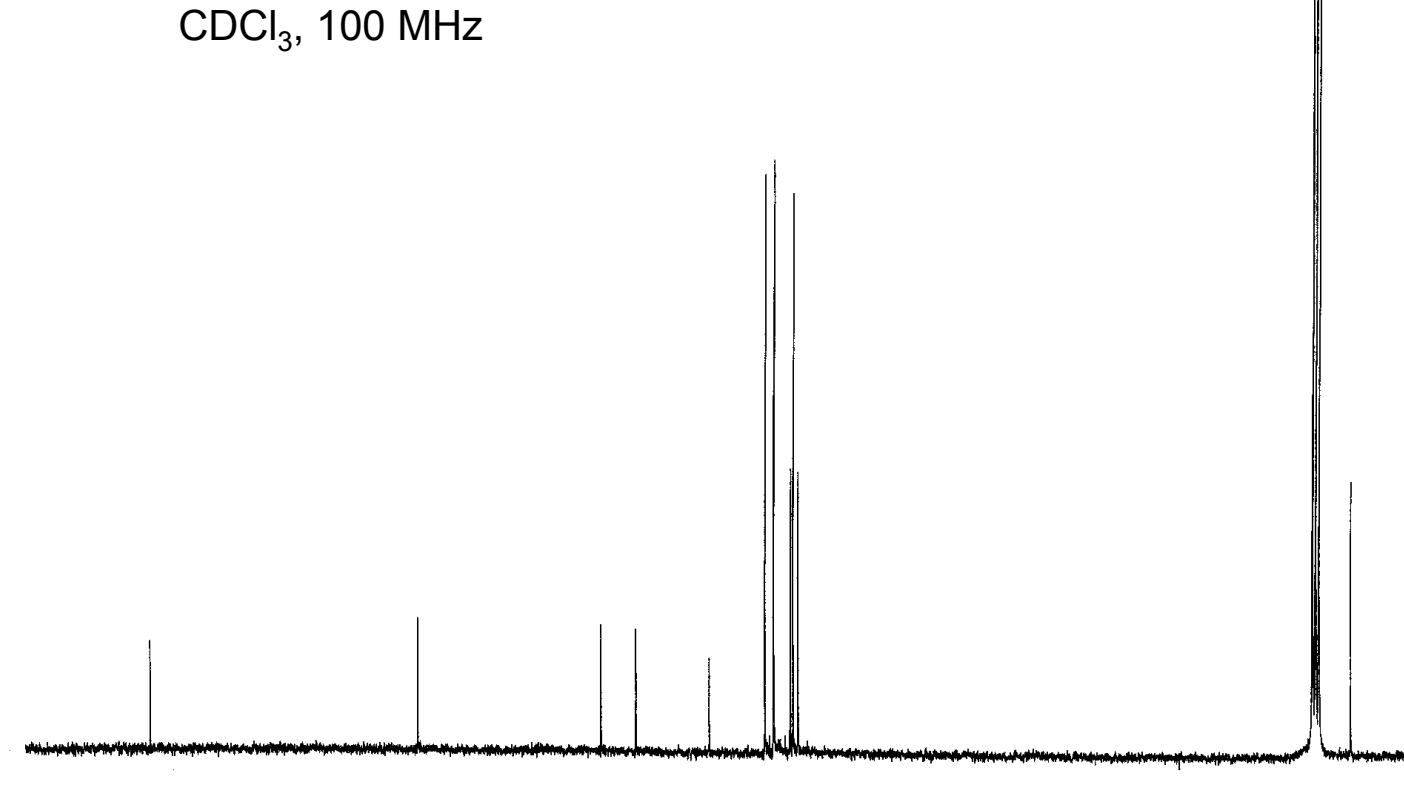

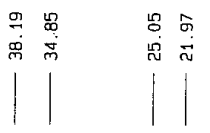

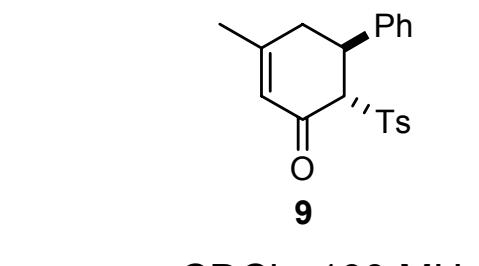




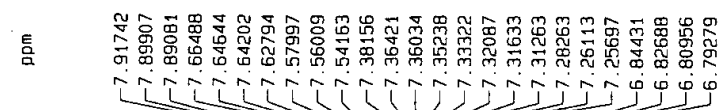

$\rightarrow-1$

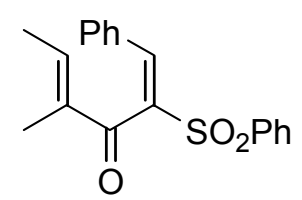

$\mathrm{CDCl}_{3}, 400 \mathrm{MHz}$

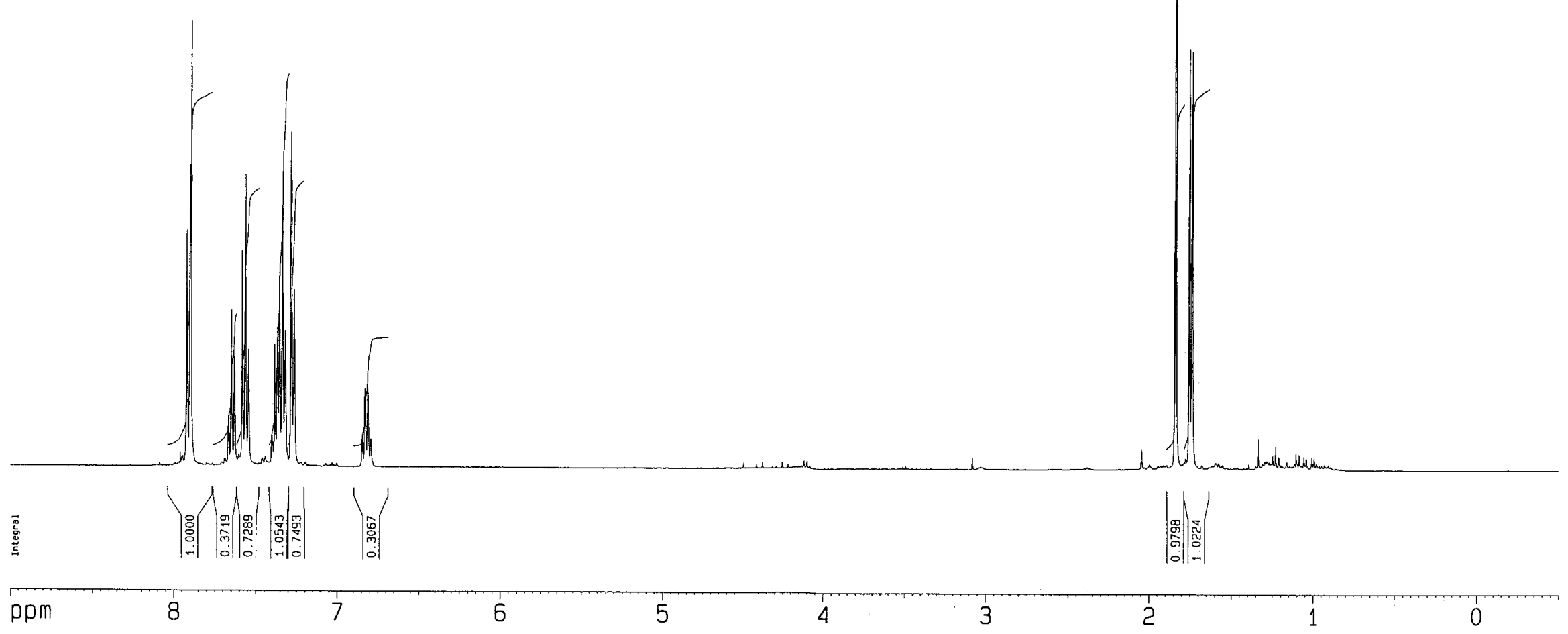


हE

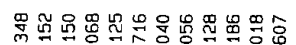

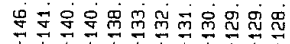

जएग

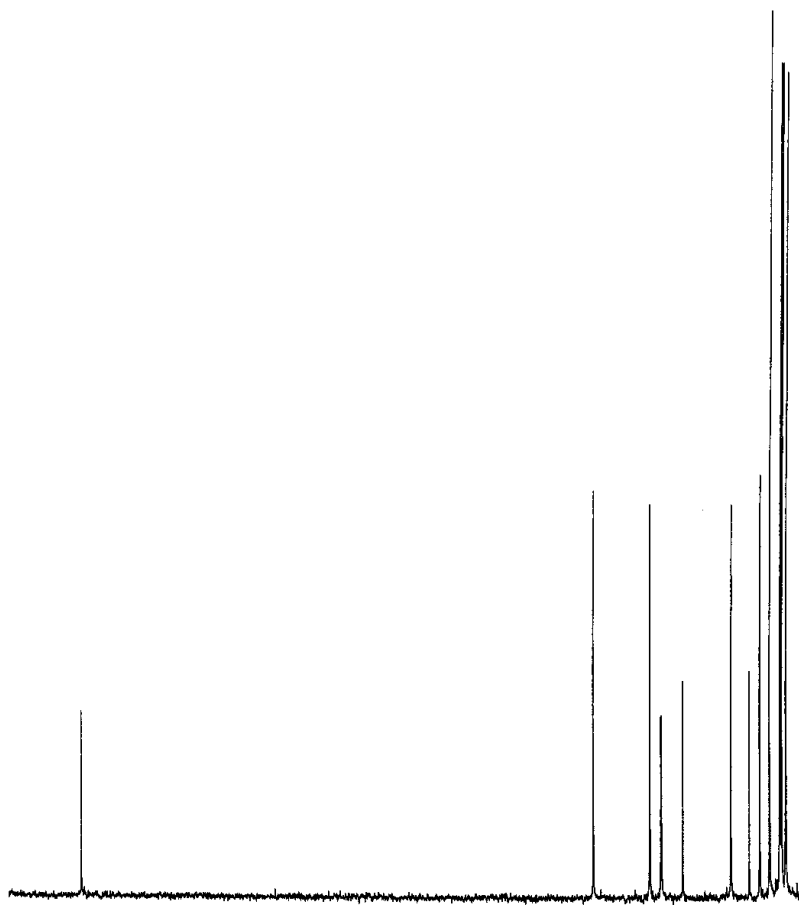

ppm

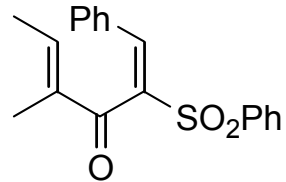

$\mathrm{CDCl}_{3}, 100 \mathrm{MHz}$ 


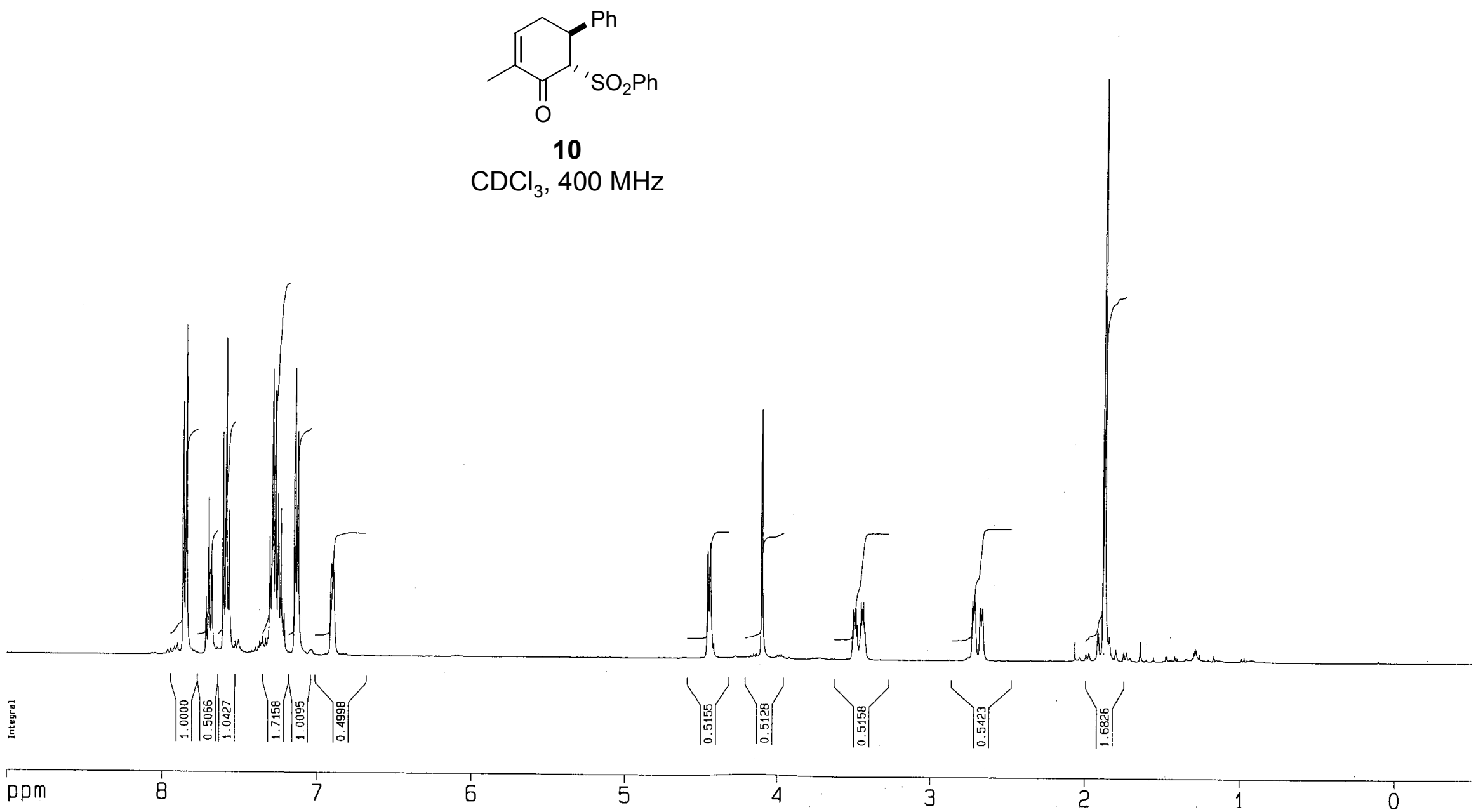




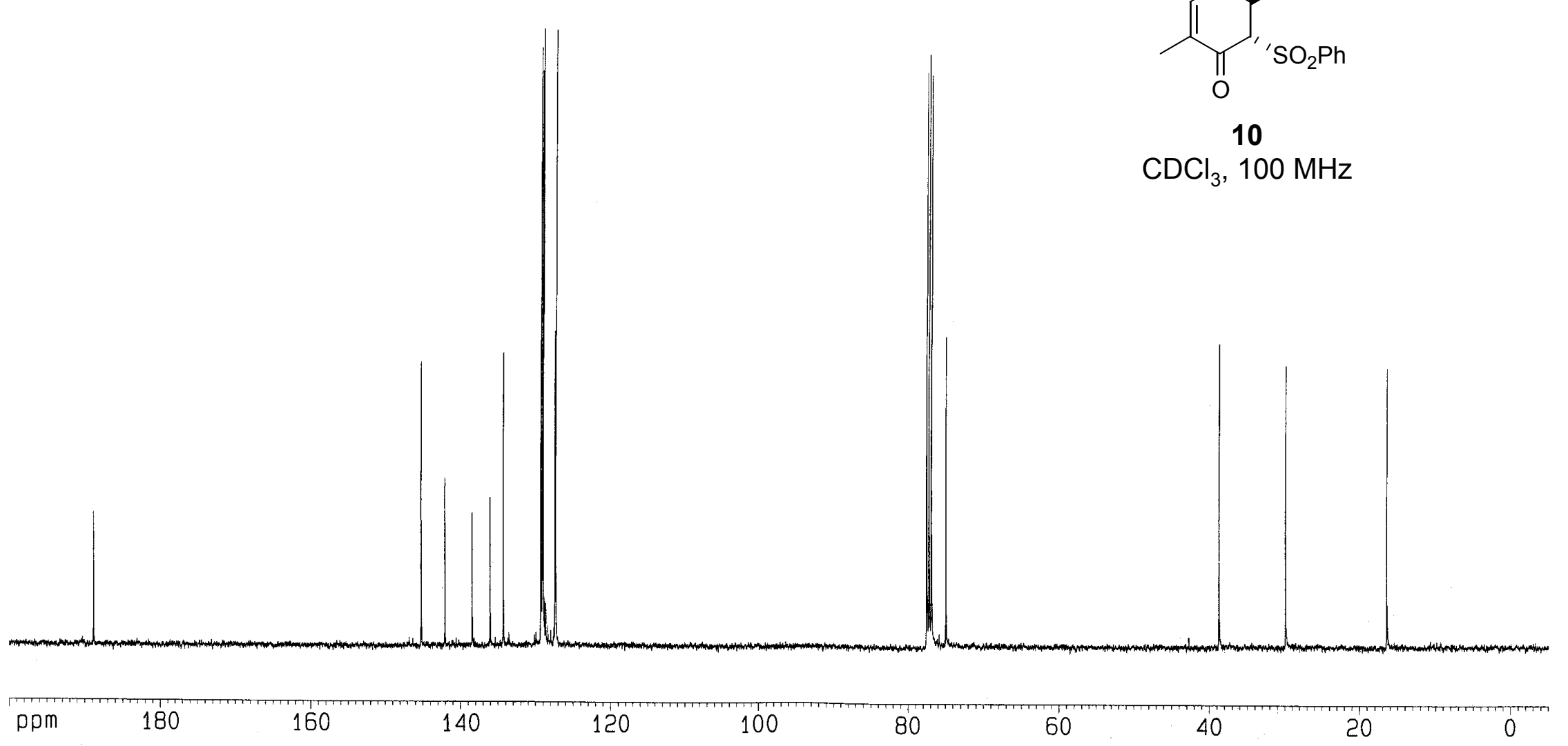



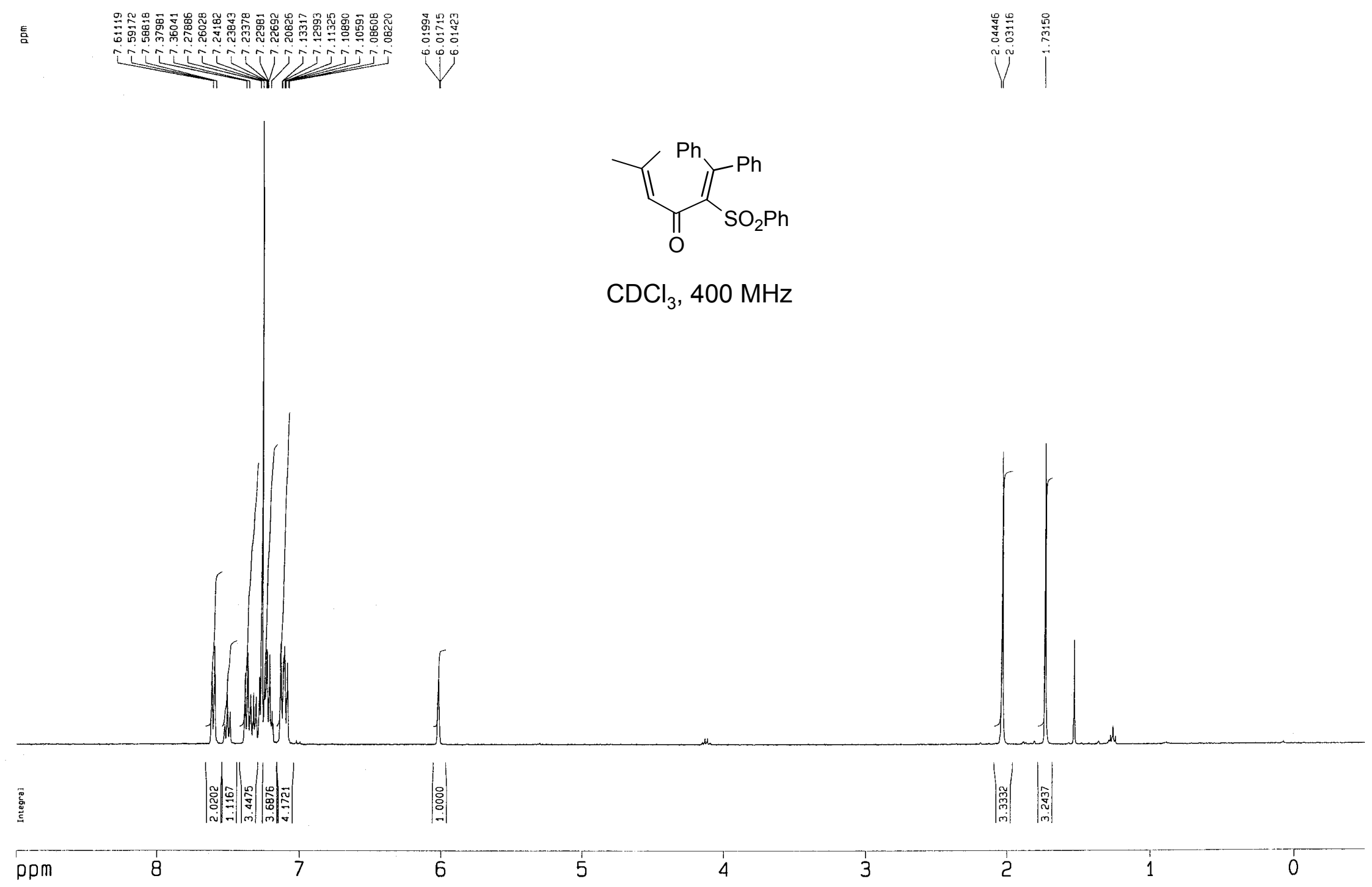


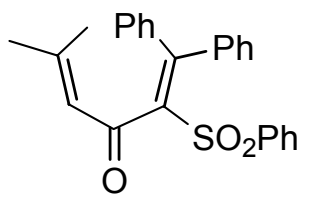

$\mathrm{CDCl}_{3}, 100 \mathrm{MHz}$

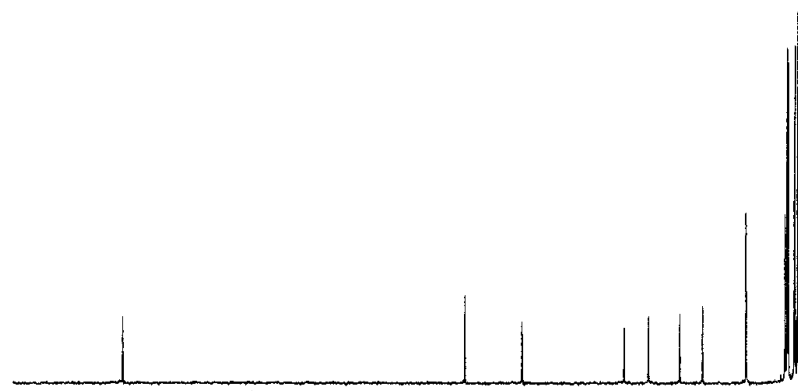




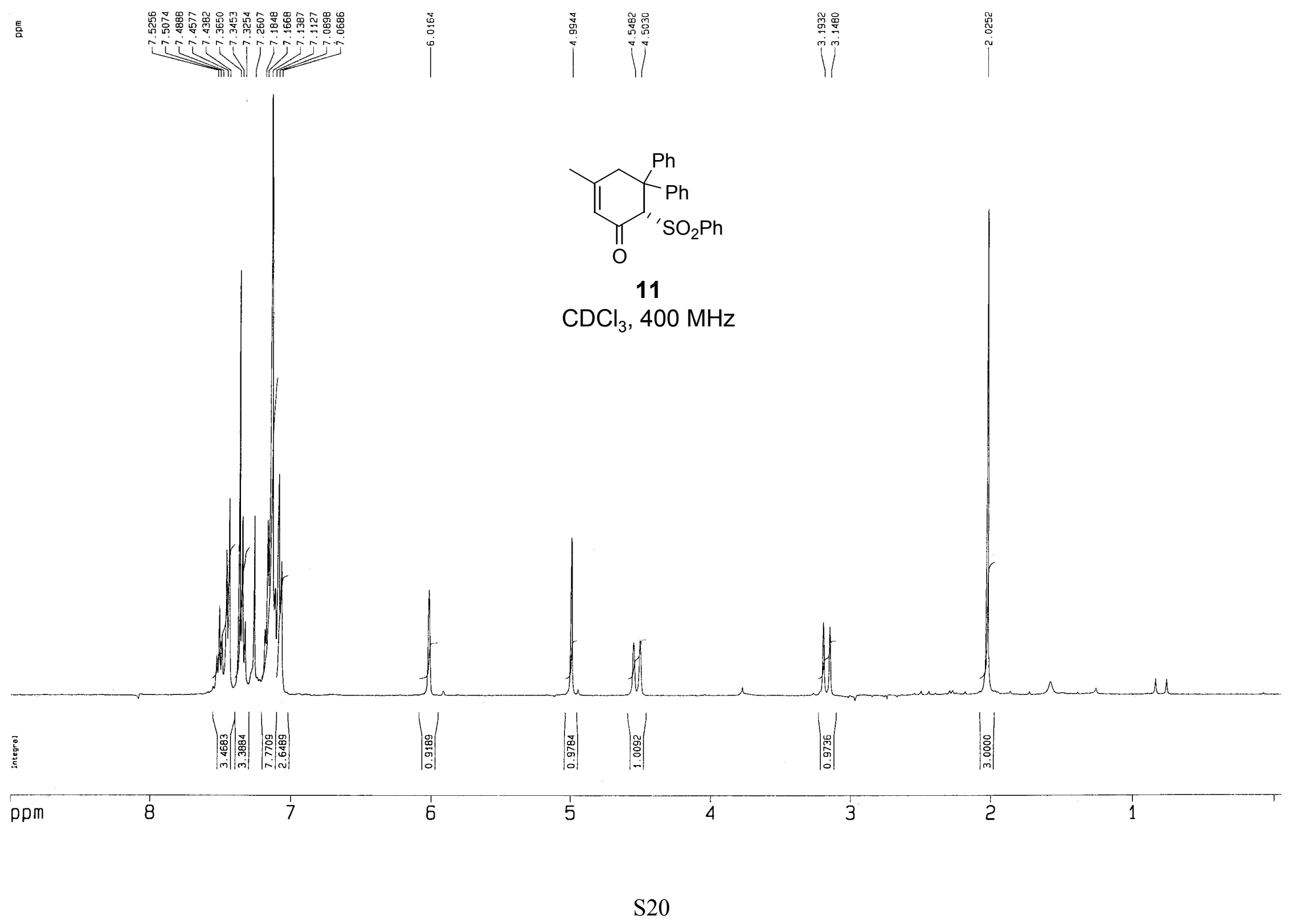




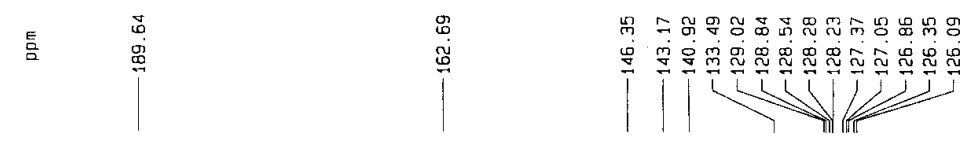

\begin{tabular}{|c|c|c|}
\hline \\
\hline \\
\hline & & 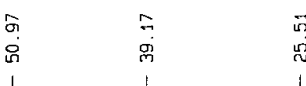 \\
\hline \\
\hline
\end{tabular}

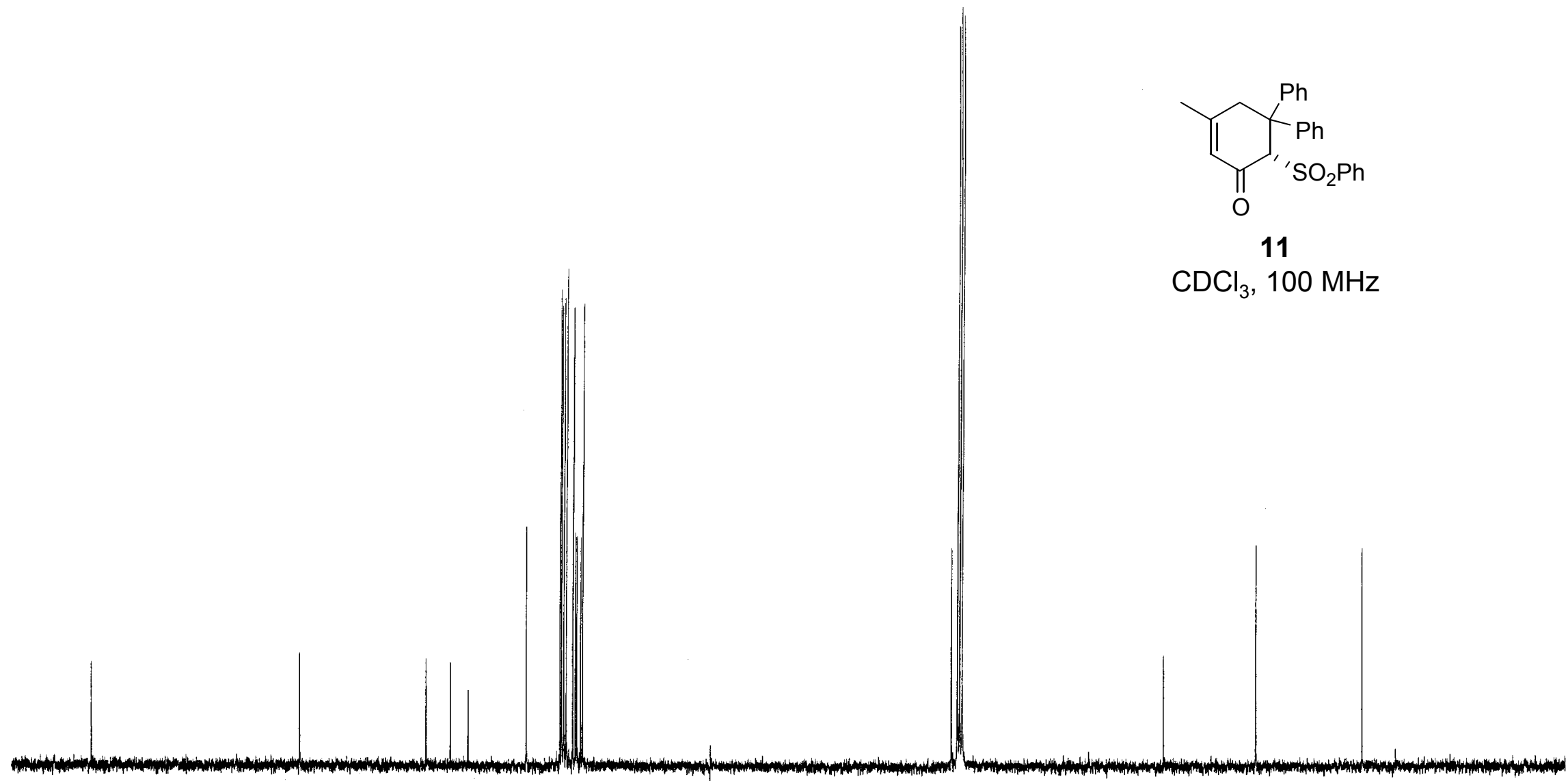



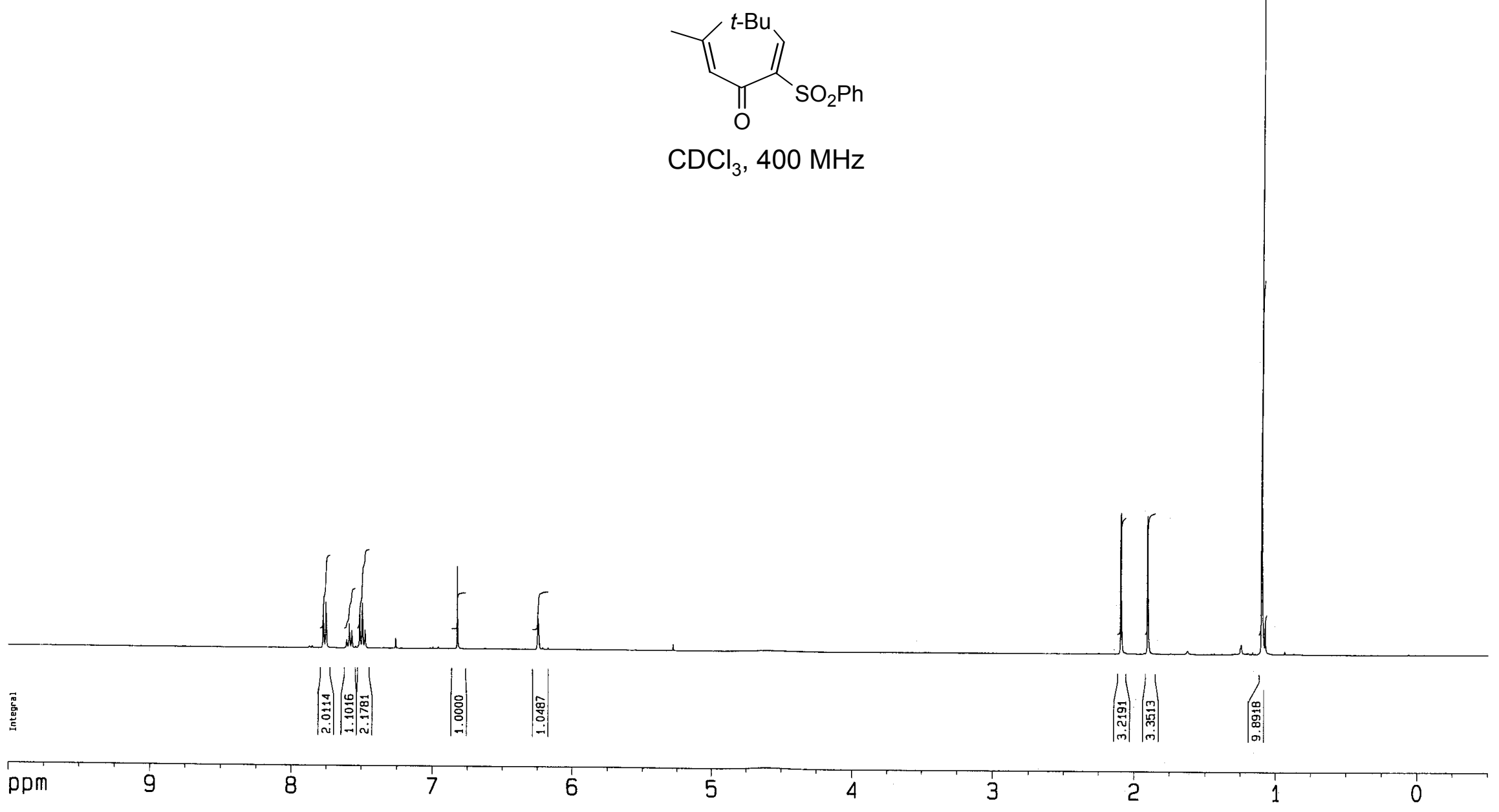

ह

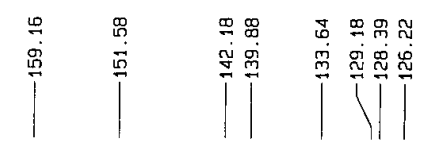
出 学 ำ
도응

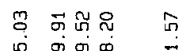
W
$|\psi| \mid$

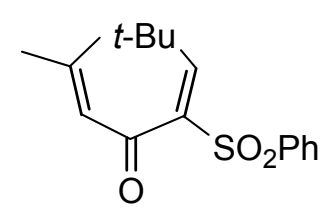

$\mathrm{CDCl}_{3}, 100 \mathrm{MHz}$

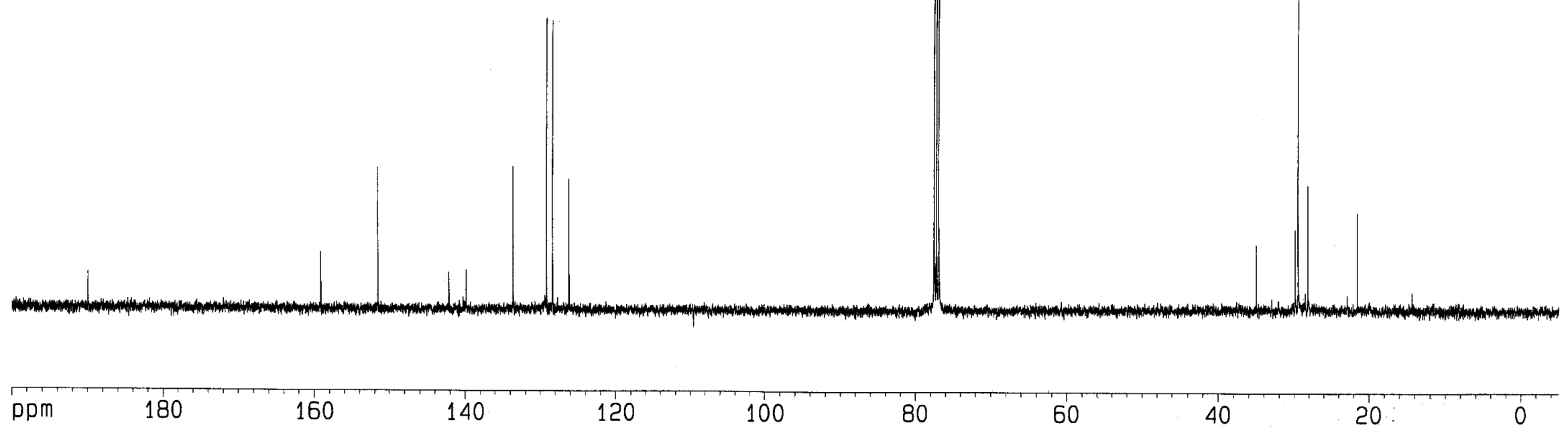




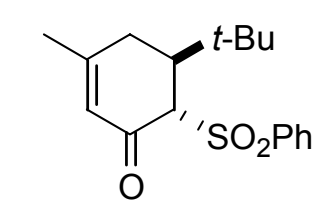

12

$\mathrm{CDCl}_{3}, 400 \mathrm{MHz}$

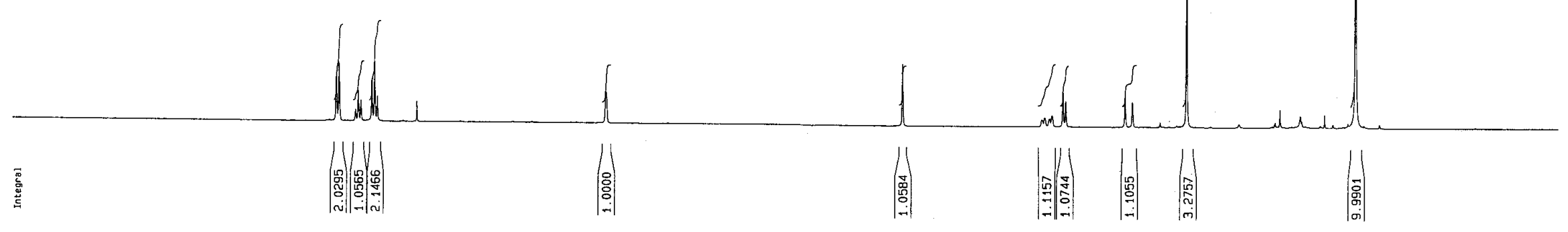




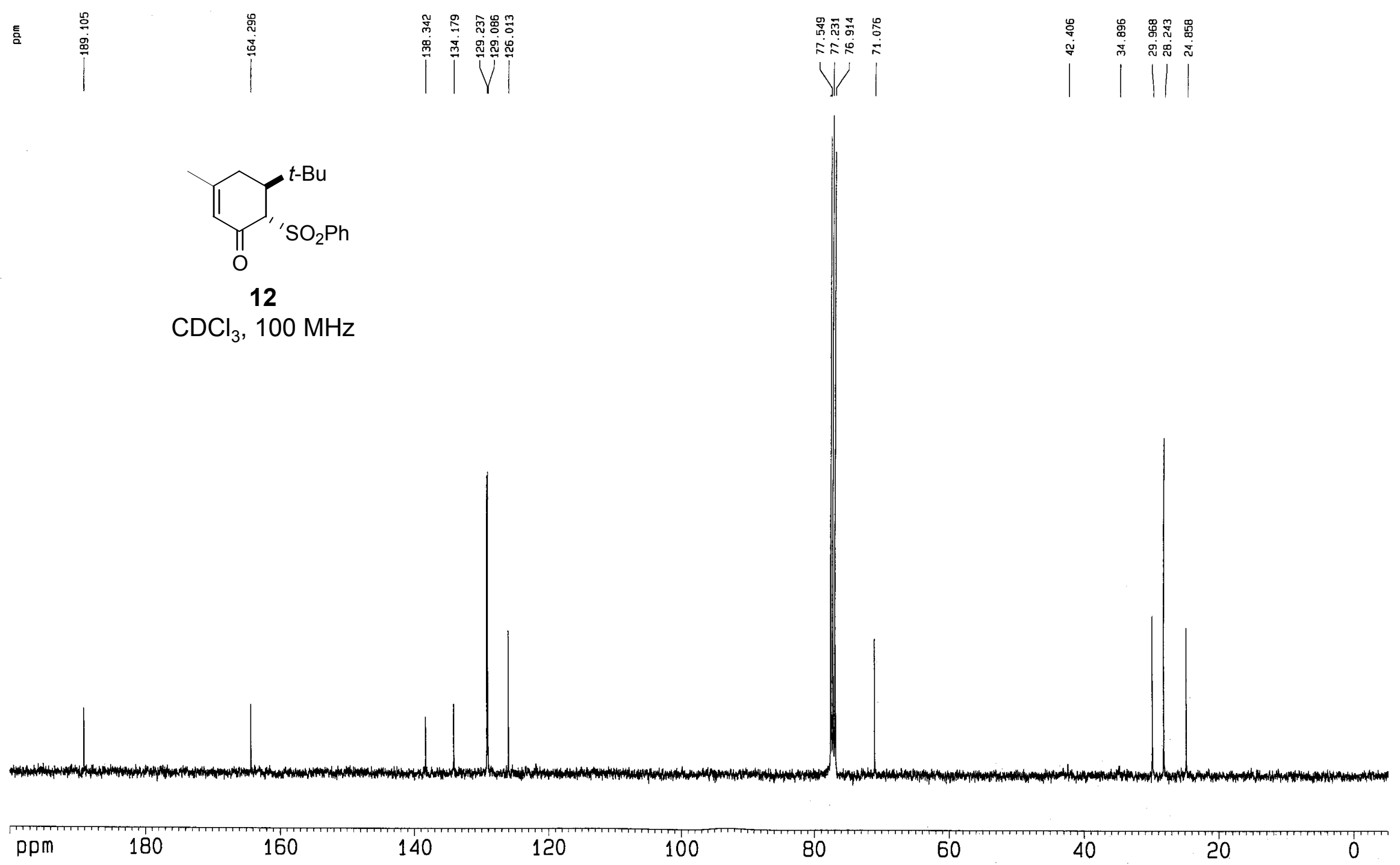


言

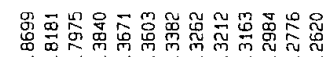

nitruinisis

$\mathrm{CDCl}_{3}, 400 \mathrm{MHz}$
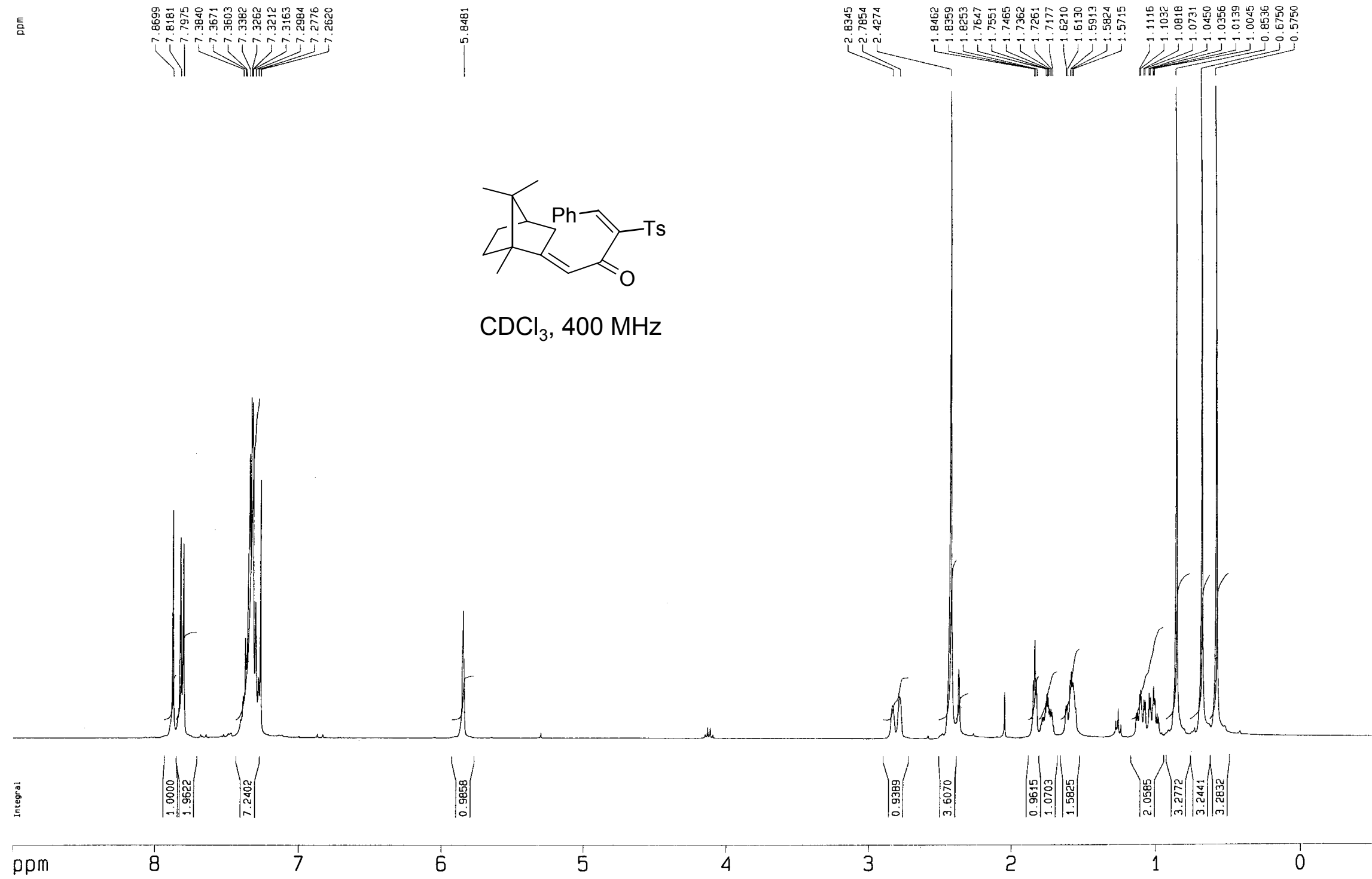

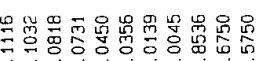

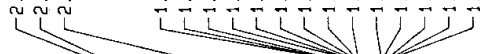

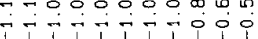




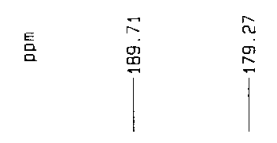

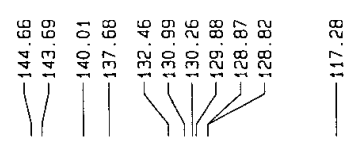

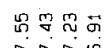

둥주은

V

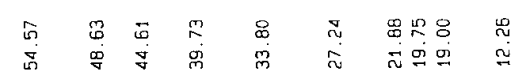

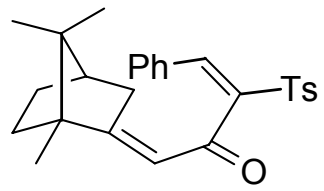

$\mathrm{CDCl}_{3}, 100 \mathrm{MHz}$

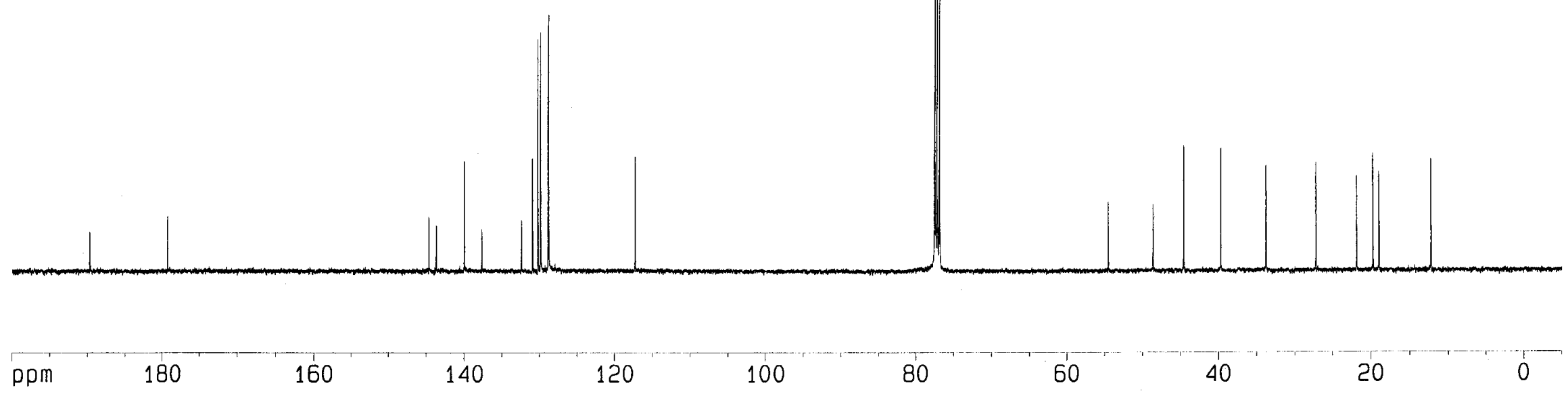




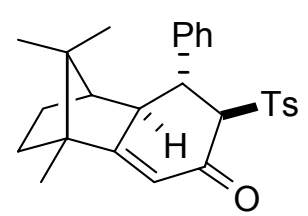

14

$\mathrm{CDCl}_{3}, 400 \mathrm{MHz}$
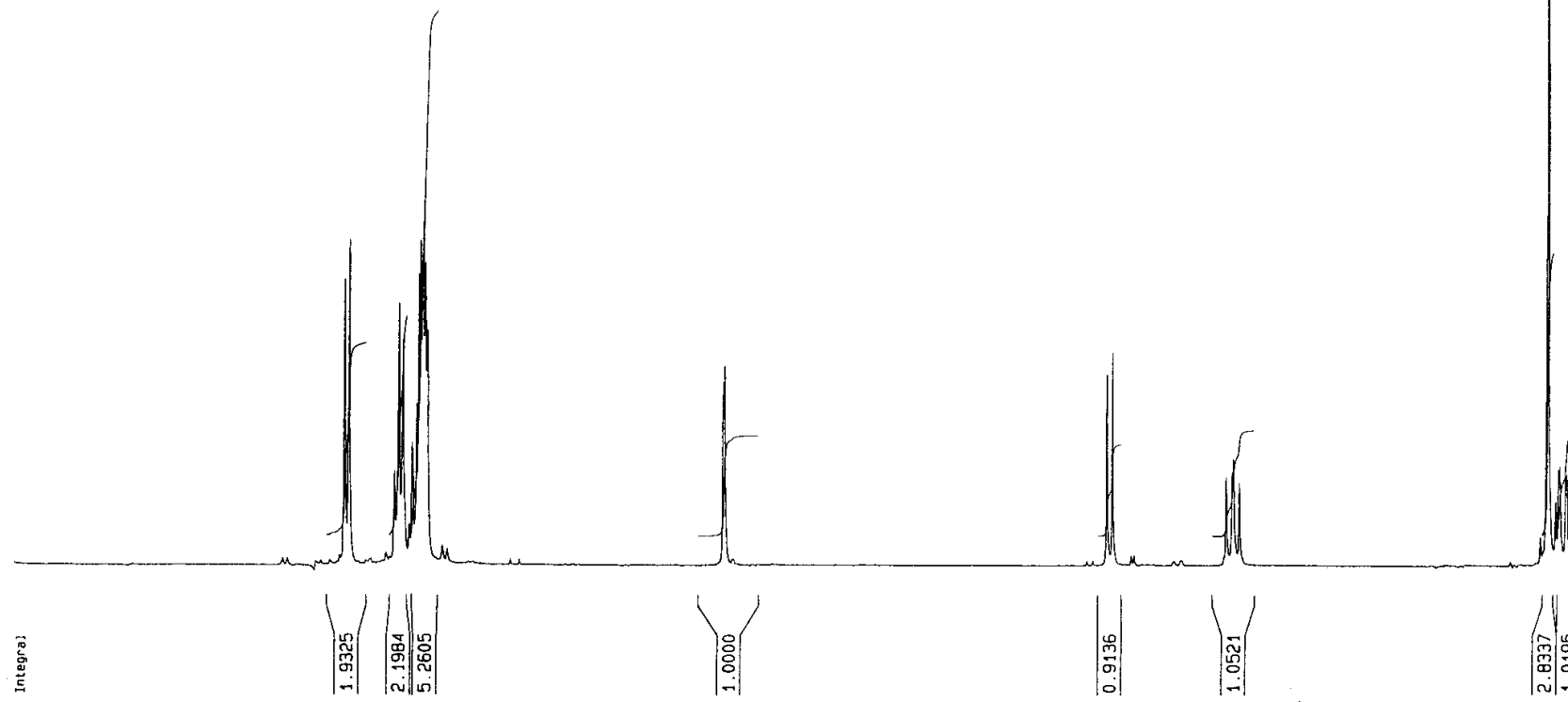
Anduld
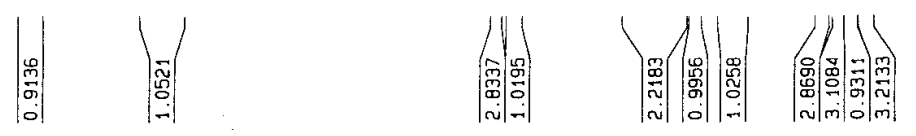

4

3

2

1 
镸

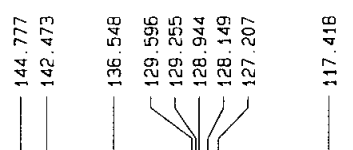

궁췅ㅎㅇ뭉

र八

y)

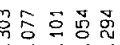

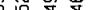

111

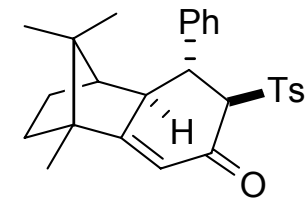

14

$\mathrm{CDCl}_{3}, 100 \mathrm{MHz}$

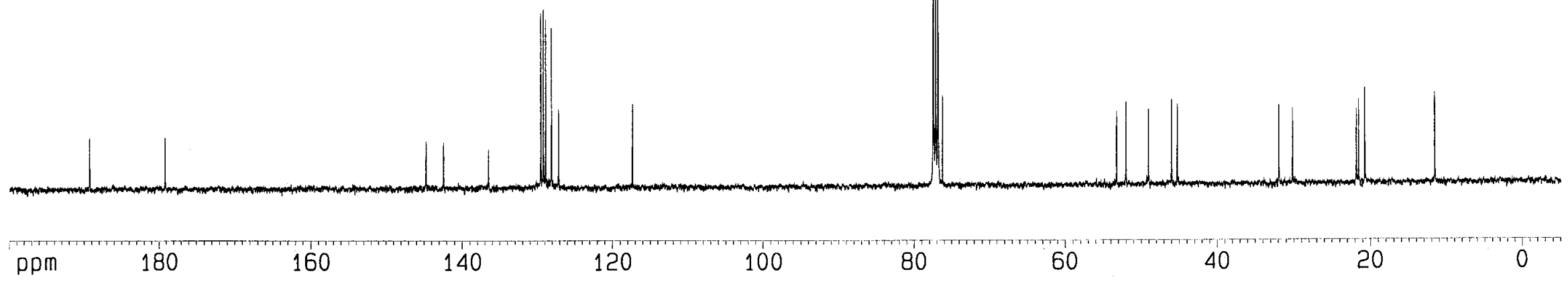




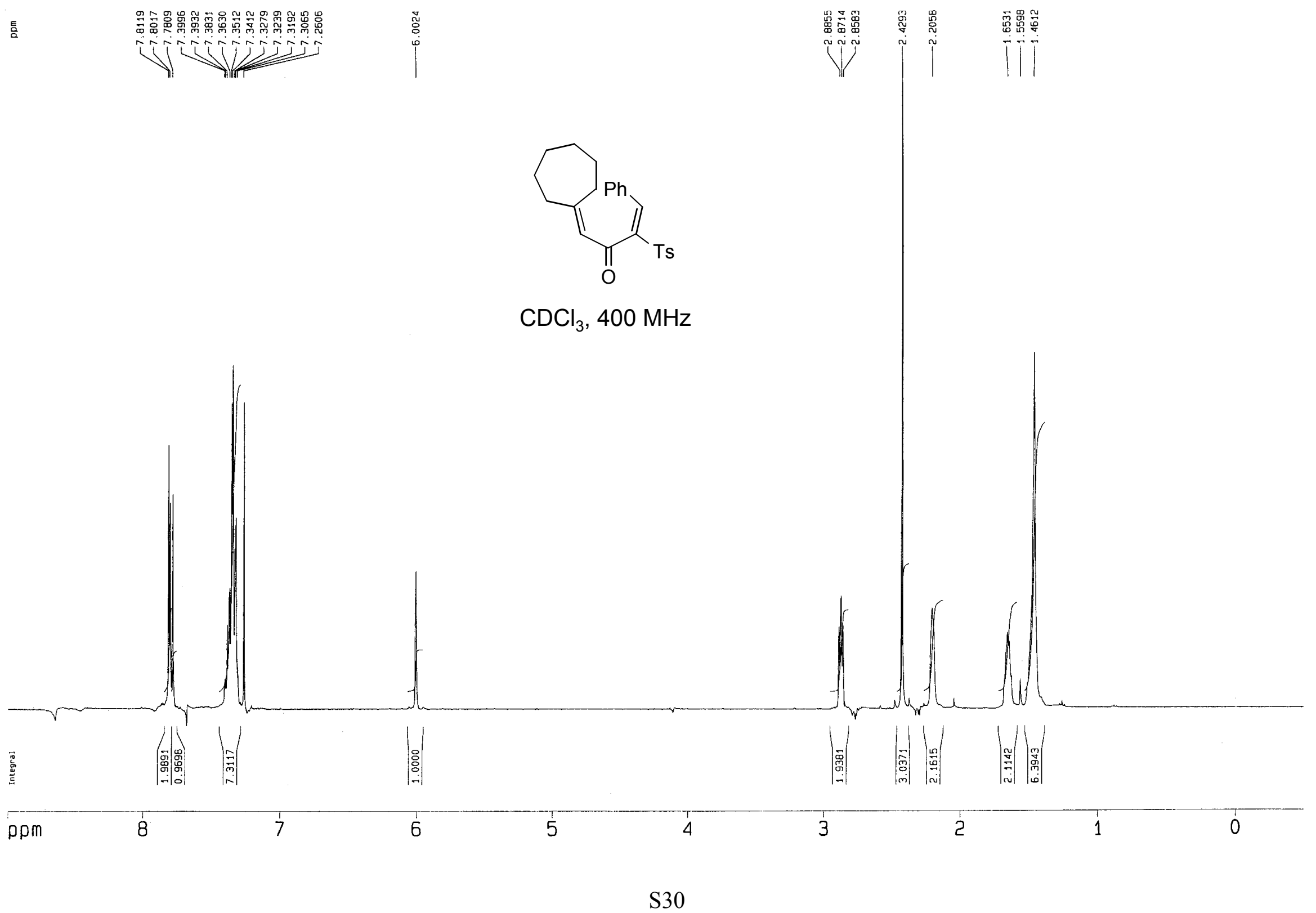


है।

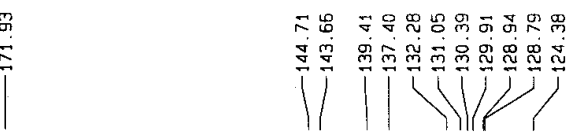

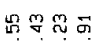

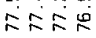

W

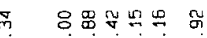

U11

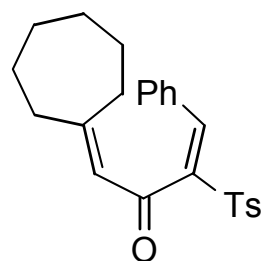

$\mathrm{CDCl}_{3}, 100 \mathrm{MHz}$ 


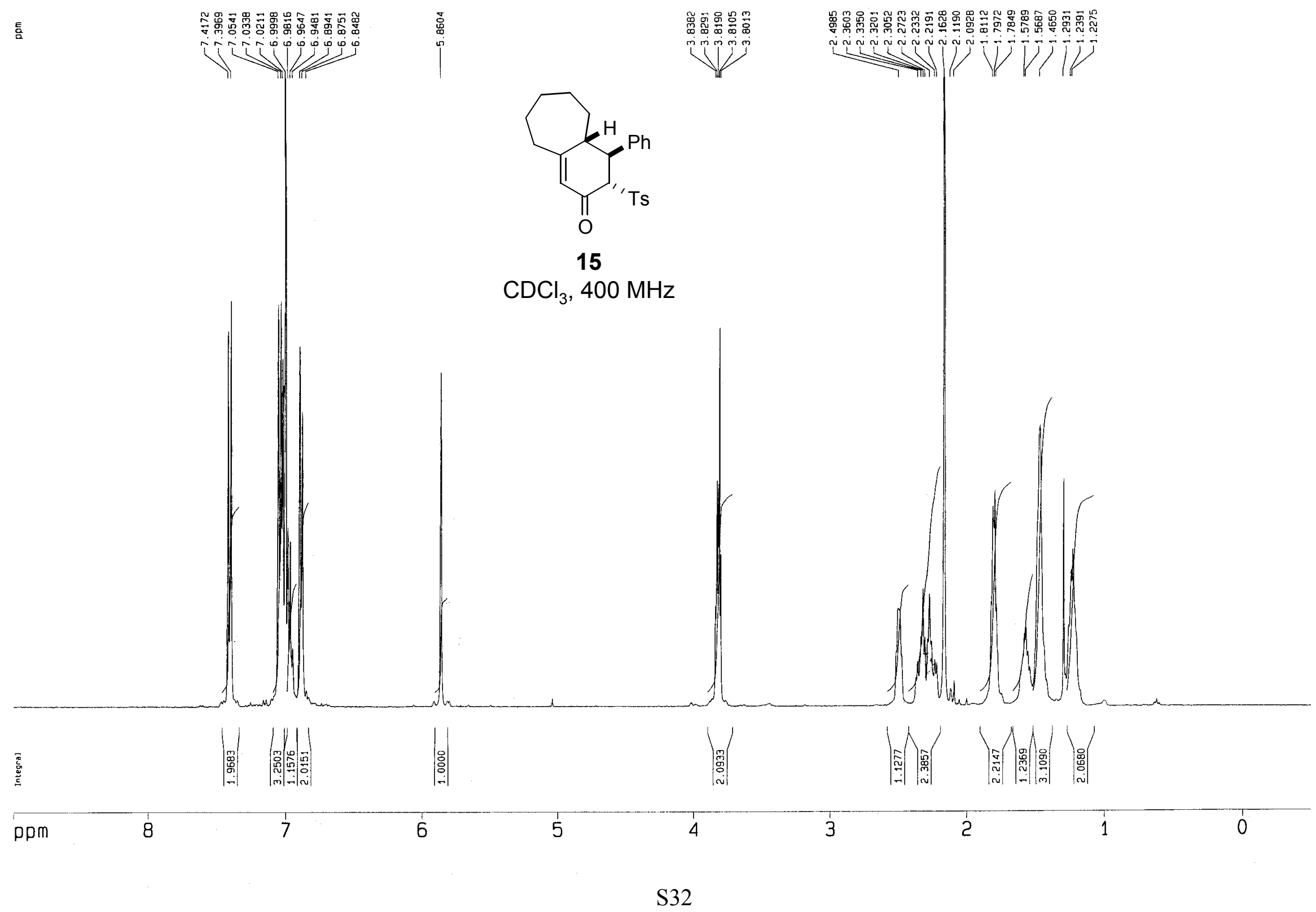




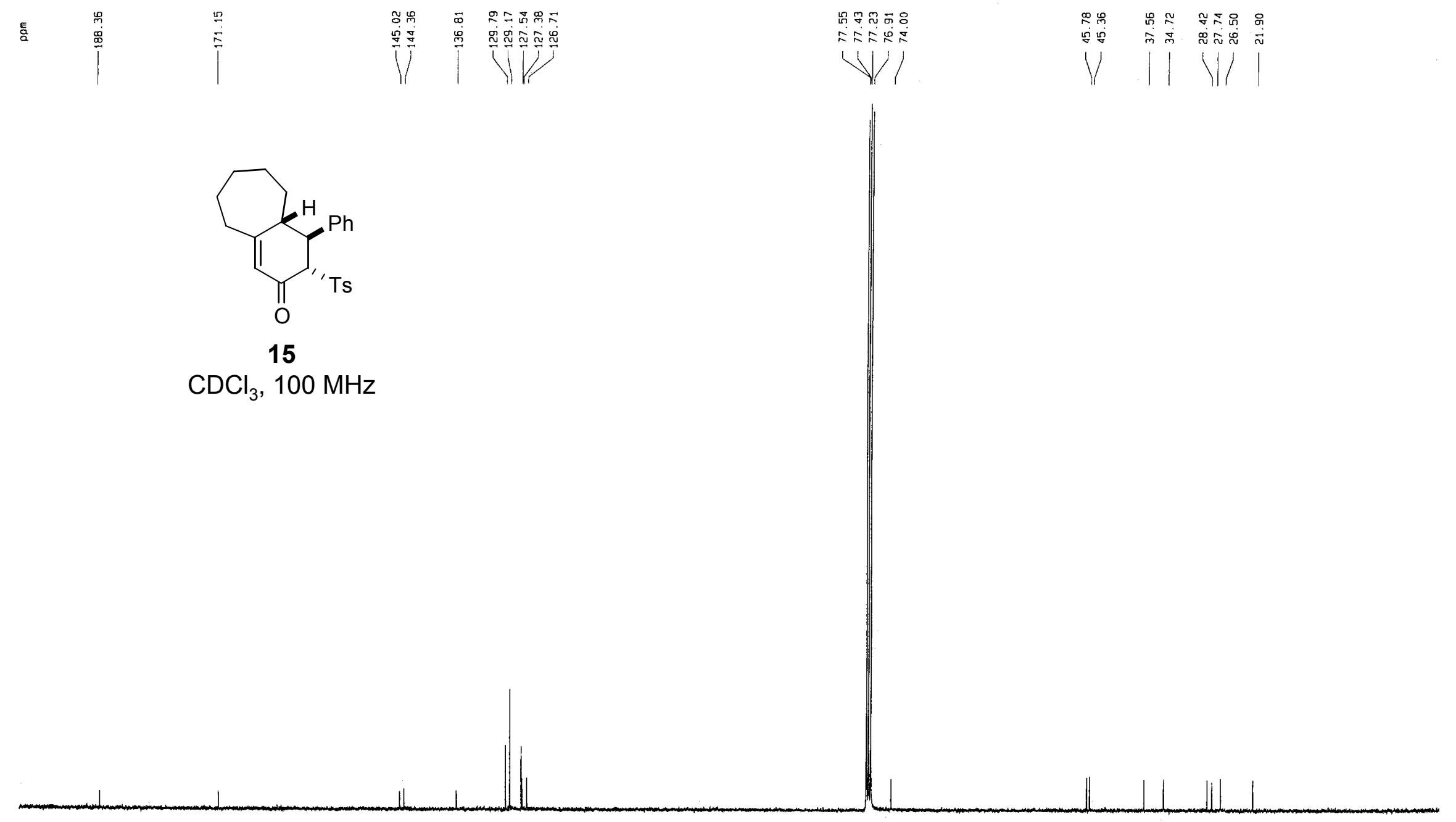


๕.

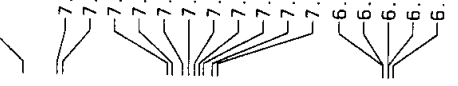

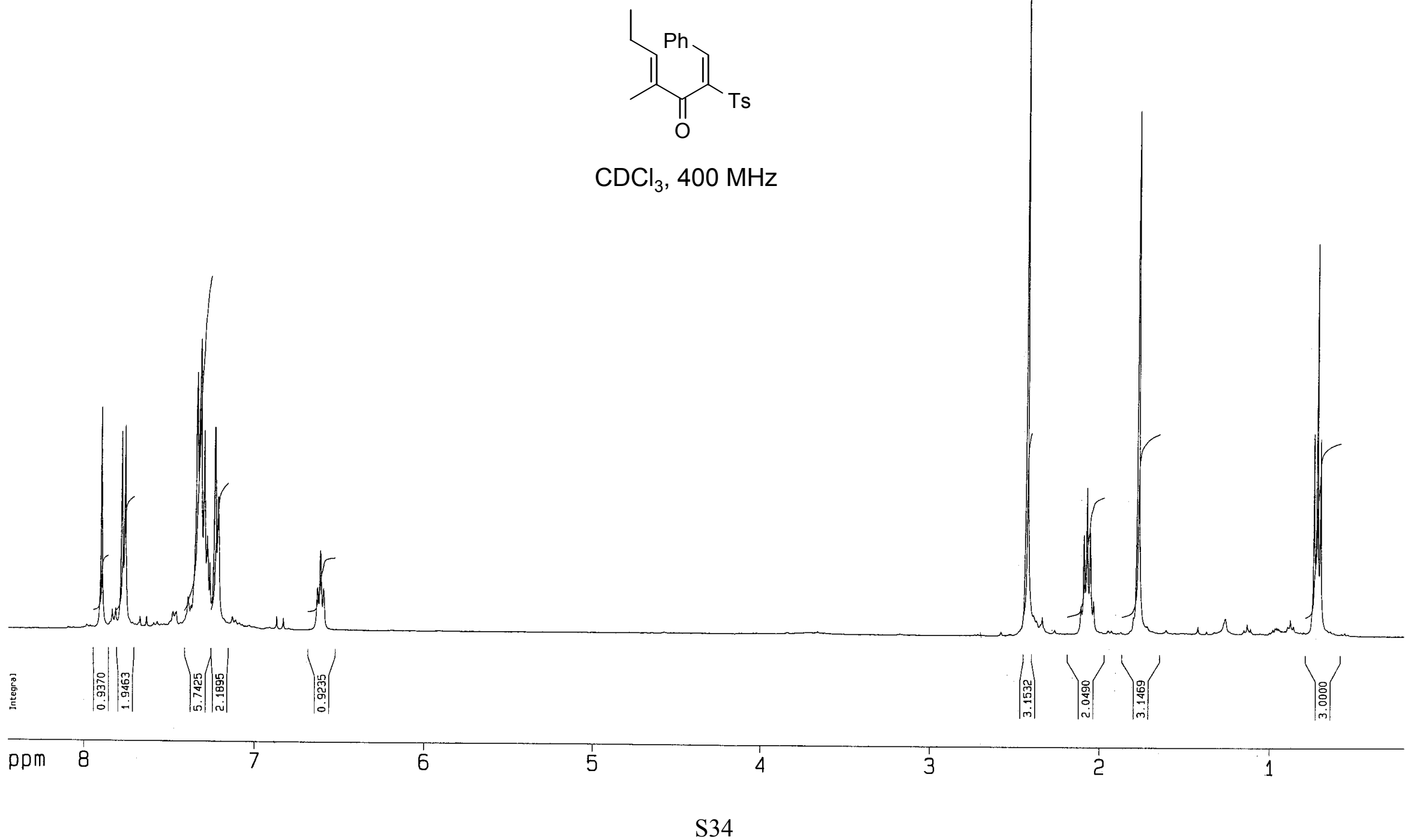




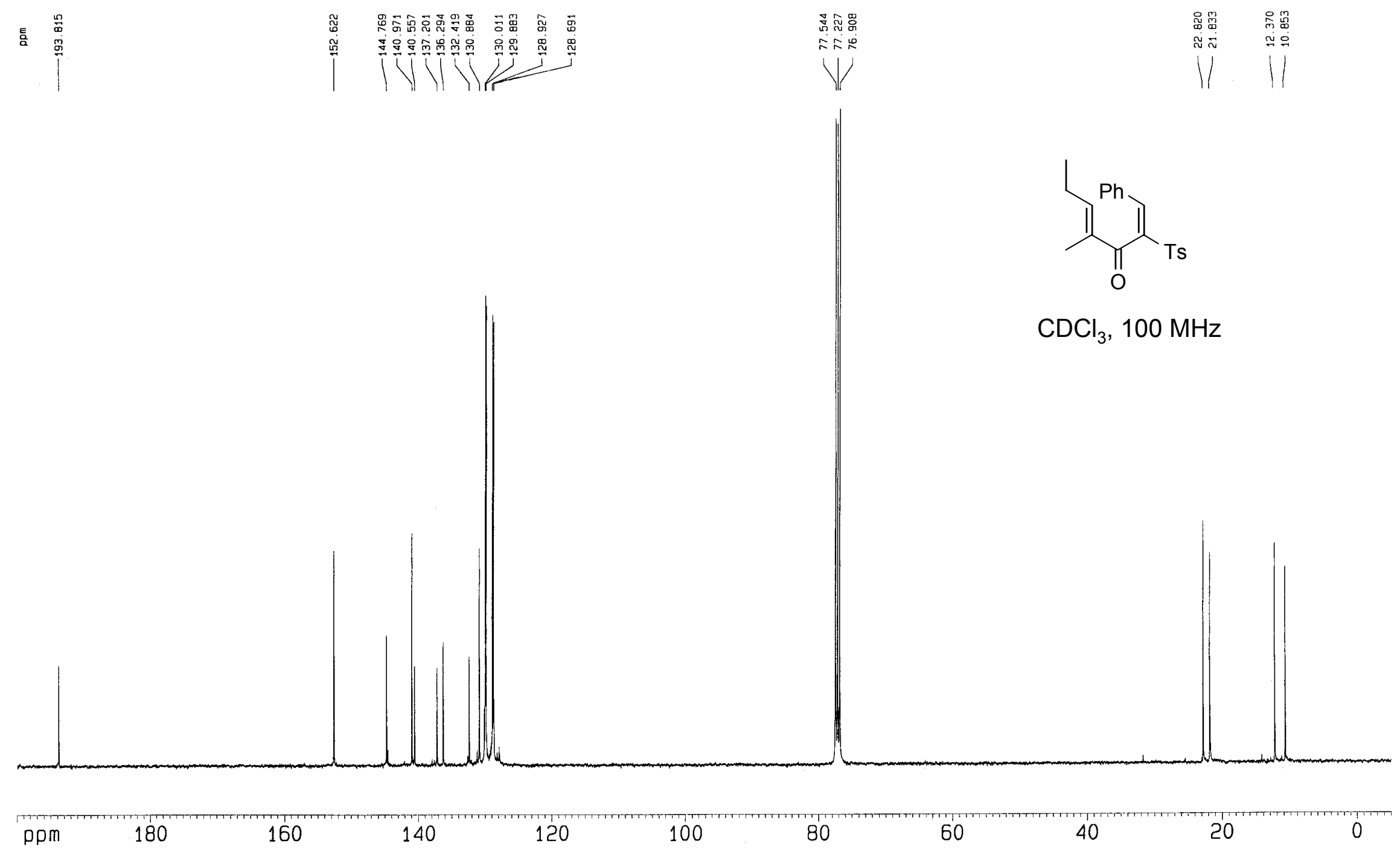




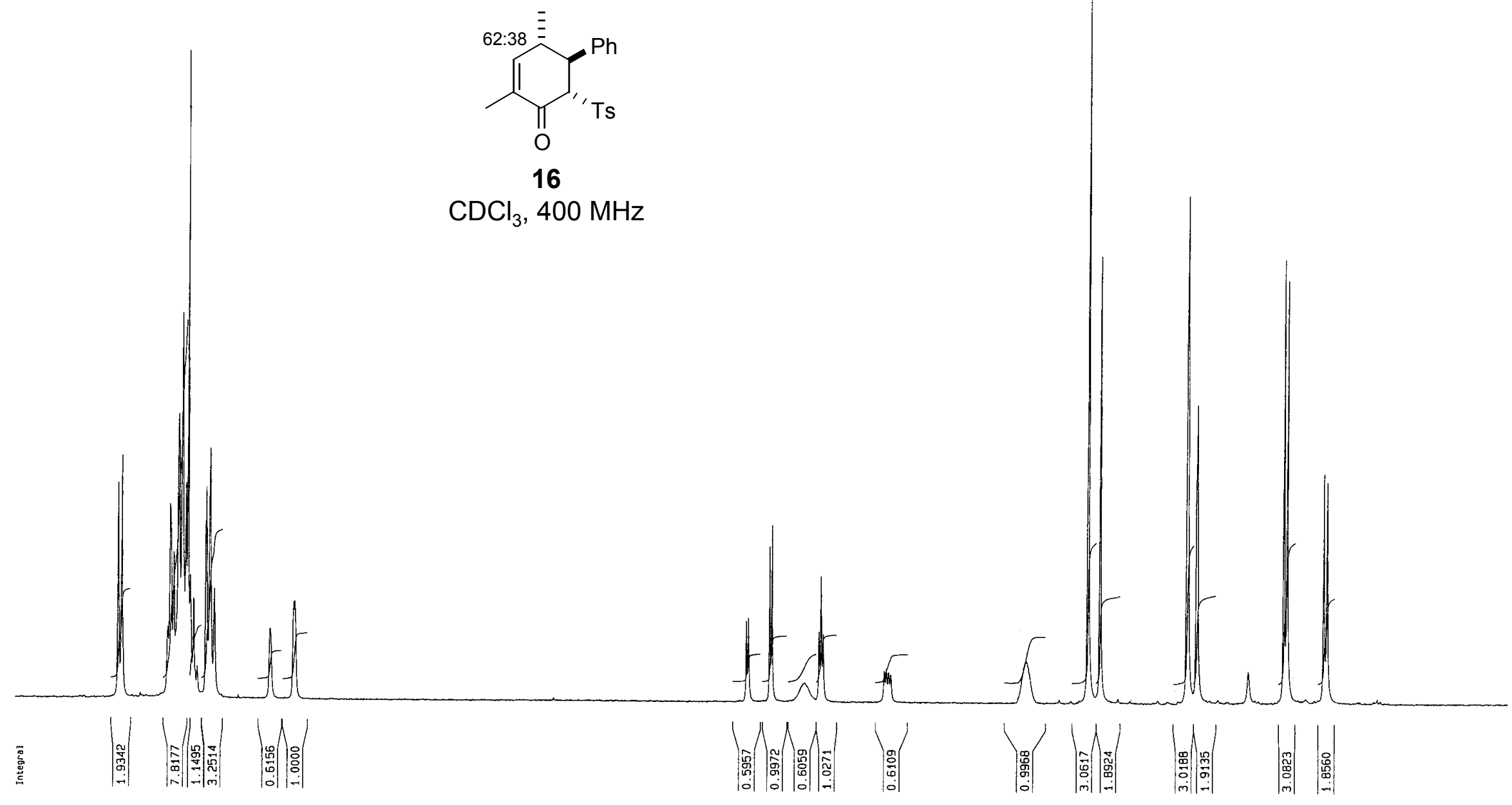

7

6

5

4

3

2

1 


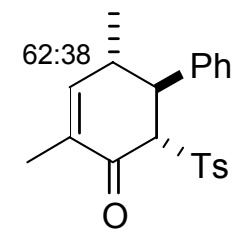

16

$\mathrm{CDCl}_{3}, 100 \mathrm{MHz}$

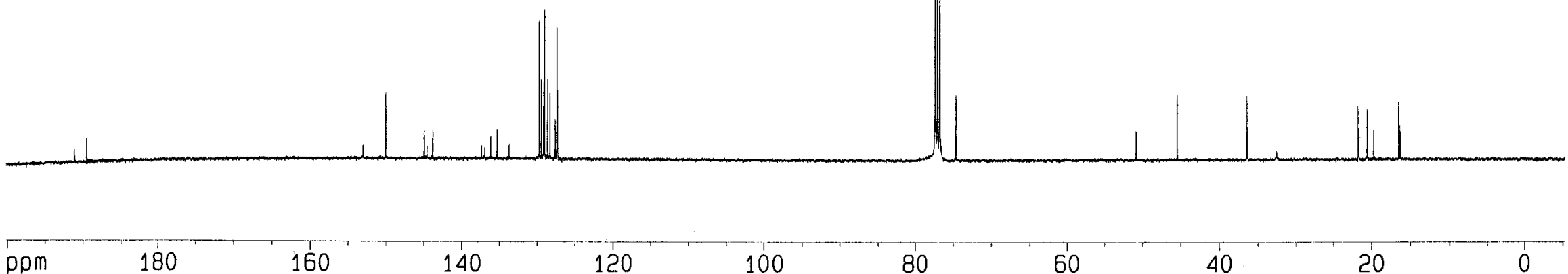




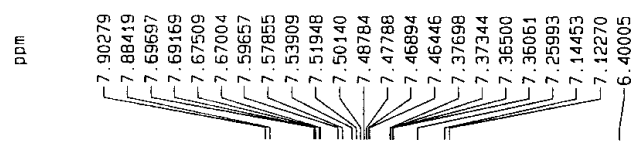
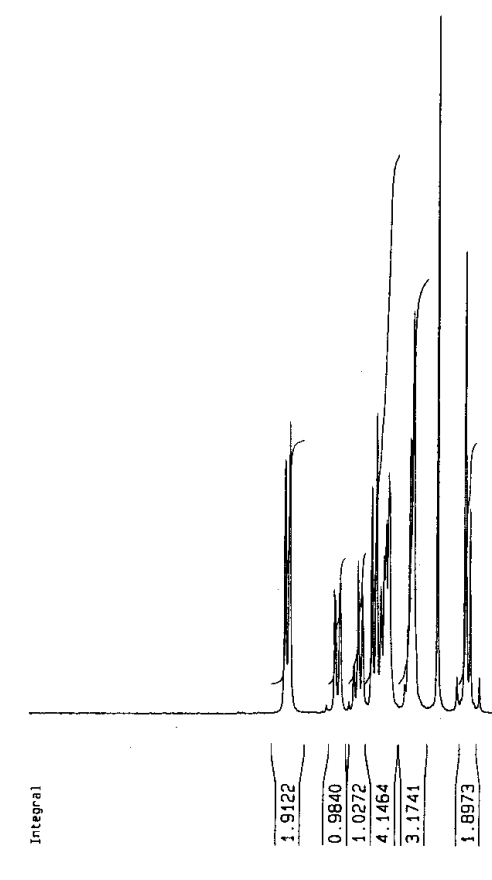

|

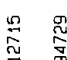

$\stackrel{\sim}{i}$

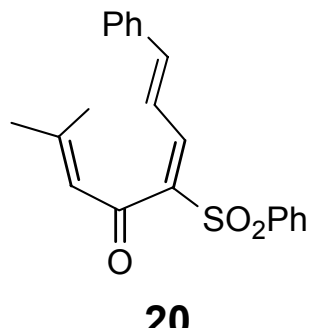
$\mathrm{CDCl}_{3}, 400 \mathrm{MHz}$

$\left|\begin{array}{l} \\ 0 \\ 0 \\ 0 \\ 0 \\ \vdots \\ \vdots\end{array}\right|$ 

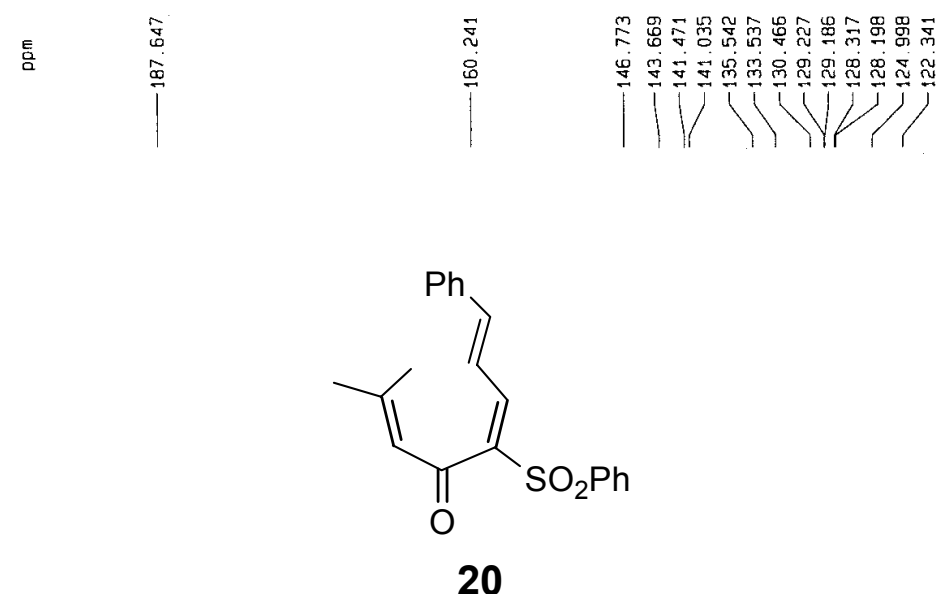

$\mathrm{CDCl}_{3}, 100 \mathrm{MHz}$

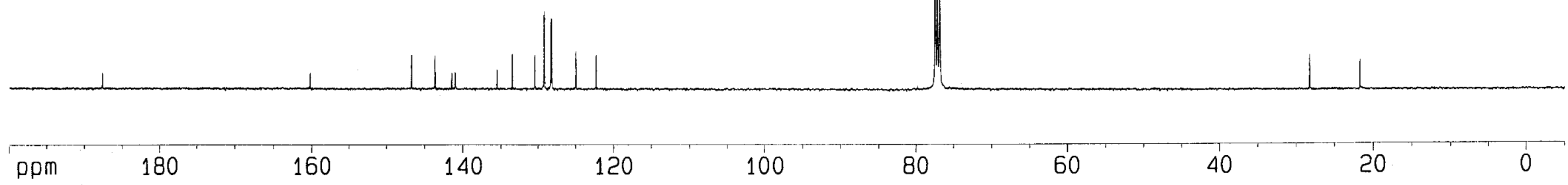




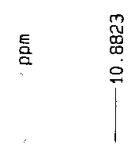

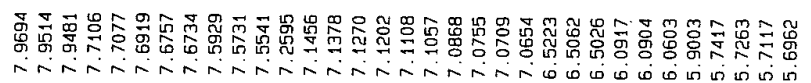

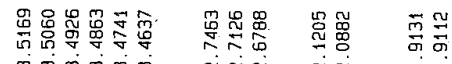

L in

$\bigcup^{1}$

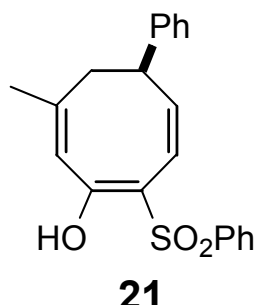

$\mathrm{CDCl}_{3}, 400 \mathrm{MHz}$

Contains about $5 \%$ of the keto form (minor peaks)

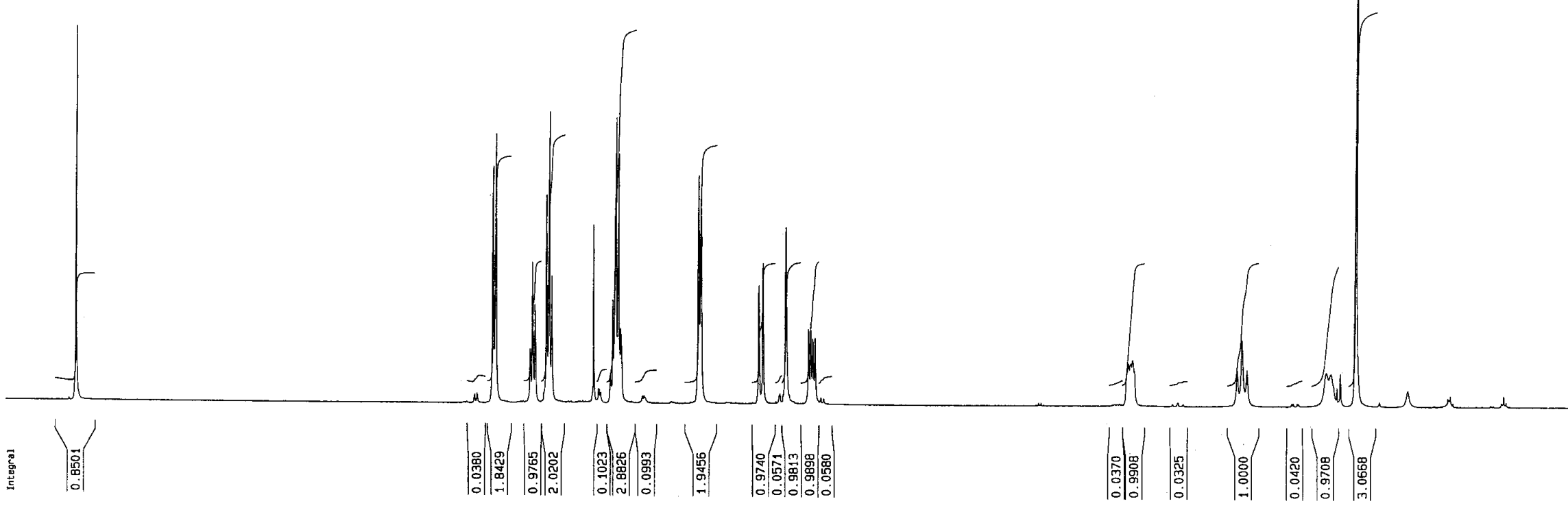

ppm

10

9

8

6

5

4

3

2 

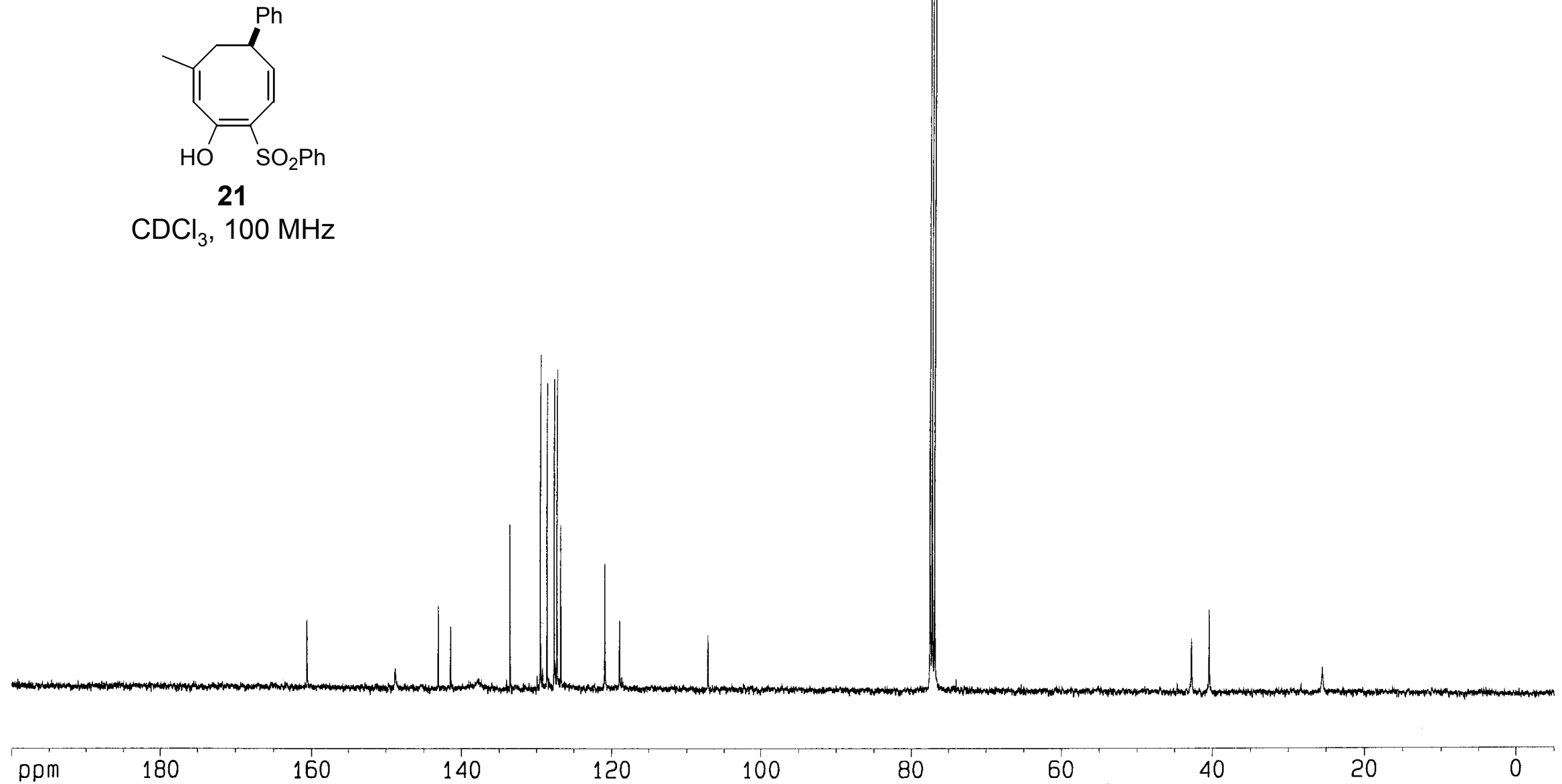
$\stackrel{\overline{5}}{\stackrel{\vec{D}}{\circ}} \stackrel{\overrightarrow{0}}{\stackrel{0}{0}}$
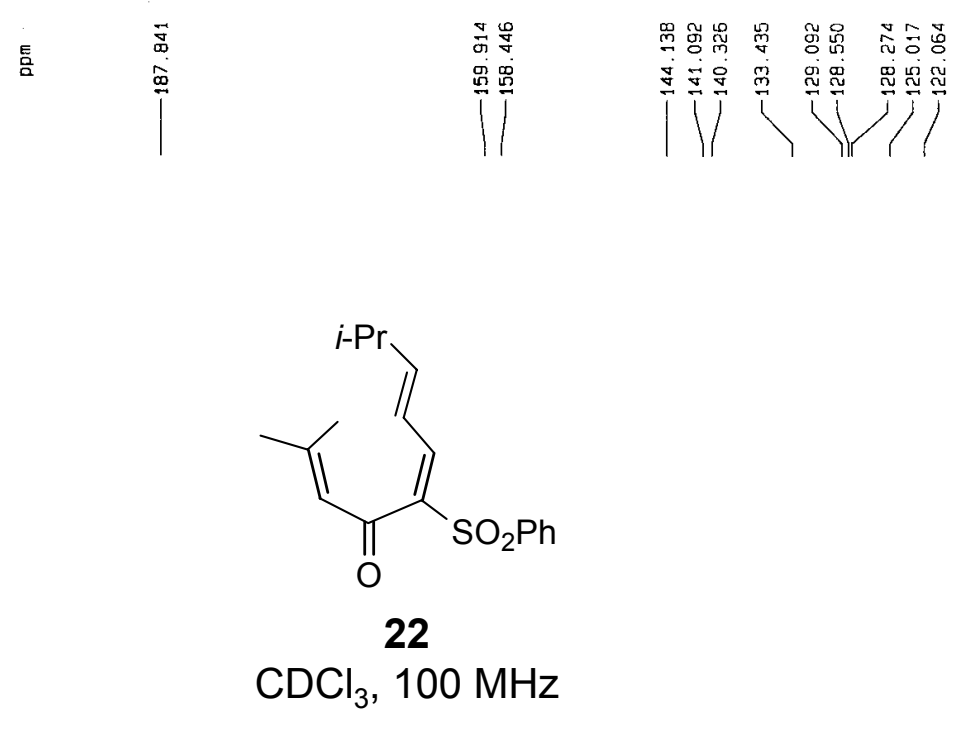

22

$\mathrm{CDCl}_{3}, 100 \mathrm{MHz}$

孚受视品

조은

W

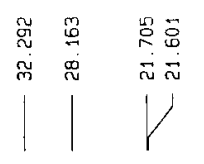

ppm

180

160

140

120

100

80

60

40

20

0

S42 


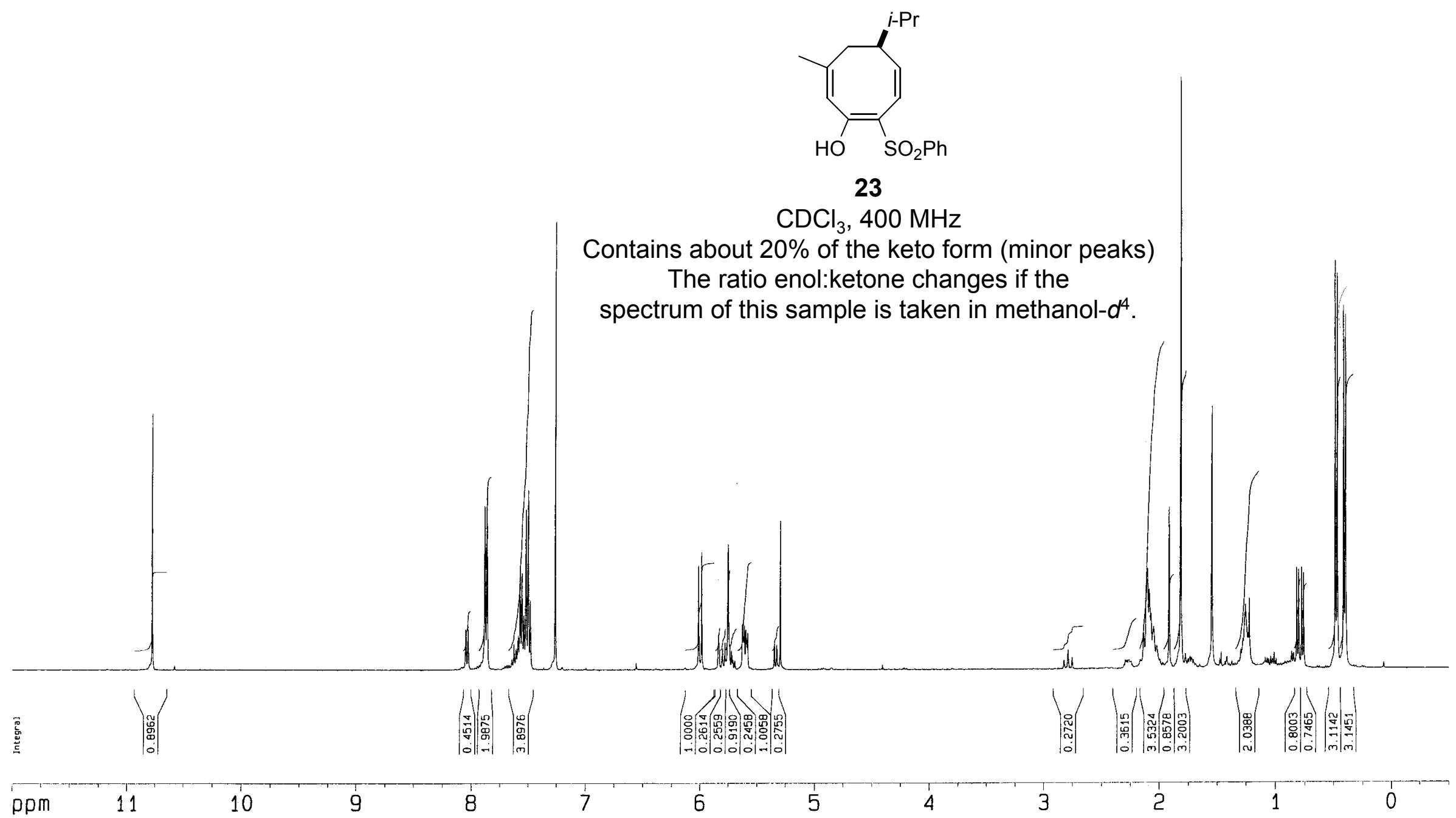

